\title{
TLR-stimulated IRAKM activates caspase-8 inflammasome in microglia and promotes neuroinflammation
}

\author{
Cun-Jin Zhang,,$^{1,2,3}$ Meiling Jiang, ${ }^{1,3,4}$ Hao Zhou, ${ }^{3}$ Weiwei Liu, ${ }^{3}$ Chenhui Wang, ${ }^{3,5,6}$ Zizhen Kang, ${ }^{3}$ Bing Han, ${ }^{3}$ Quanri Zhang, ${ }^{3}$ \\ Xing Chen, ${ }^{3}$ Jianxin Xiao, ${ }^{3}$ Amanda Fisher, ${ }^{7}$ William J. Kaiser, ${ }^{7}$ Masanori A. Murayama, ${ }^{8,9}$ Yoichiro Iwakura, ${ }^{9}$ Ji Gao, ${ }^{10}$ \\ Julie Carman, ${ }^{10}$ Ashok Dongre, ${ }^{10}$ George Dubyak, ${ }^{11}$ Derek W. Abbott, ${ }^{12}$ Fu-Dong Shi, ${ }^{2,13}$ Richard M. Ransohoff, ${ }^{14}$ and Xiaoxia Li ${ }^{3}$ \\ ${ }^{1}$ Medical School of Nanjing University, Nanjing, Jiangsu, China. ${ }^{2}$ Department of Neurology, Tianjin Medical University General Hospital, Tianjin, China. ${ }^{3}$ Department of Immunology, Lerner Research Institute, \\ Cleveland Clinic, Cleveland, Ohio, USA. ${ }^{4}$ Institute of Radiation Medicine, Chinese Academy of Medical Sciences and Peking Union Medical College, Tianjin, China. ${ }^{5}$ Key Laboratory of Molecular Biophysics \\ of the Ministry of Education, College of Life Science and Technology, Huazhong University of Science and Technology, Wuhan, China. ${ }^{6}$ Wuhan Institute of Biotechnology, Wuhan, China. ${ }^{7}$ Department of \\ Microbiology and Immunology, Emory Vaccine Center, Atlanta, Georgia, USA. ${ }^{8}$ Department of Immunology and Medicine, St. Marianna University School of Medicine, Kawasaki, Japan. ${ }^{9}$ Research Institute \\ for Biomedical Sciences, Tokyo University of Science, Yamazaki, Noda, Japan. ${ }^{10}$ Discovery Biology, Bristol-Myers Squibb, Princeton, New Jersey, USA. "Department of Physiology and Biophysics, Case Western \\ Reserve University School of Medicine, Cleveland, Ohio, USA. ${ }^{12}$ Department of Pathology, Case Western Reserve University/University Hospitals Case Medical Center, Cleveland, Ohio, USA. ${ }^{13}$ Department of \\ Neurology, Barrow Neurological Institute, St. Joseph's Hospital and Medical Center, Phoenix, Arizona, USA. ${ }^{14}$ Department of Cell Biology, Harvard Medical School, Boston, Massachusetts, USA
}

\begin{abstract}
NLRP3 inflammasome plays a critical spatiotemporal role in the pathogenesis of experimental autoimmune encephalomyelitis (EAE). This study reports a mechanistic insight into noncanonical NLRP3 inflammasome activation in microglia for the effector stage of EAE. Microglia-specific deficiency of ASC (apoptosis-associated speck-like protein containing a C-terminal caspase-activation and recruitment [CARD] domain) attenuated T cell expansion and neutrophil recruitment during EAE pathogenesis. Mechanistically, TLR stimulation led to IRAKM-caspase-8-ASC complex formation, resulting in the activation of caspase- 8 and IL-1 $\beta$ release in microglia. Noncanonical inflammasome-derived IL- $1 \beta$ produced by microglia in the CNS helped to expand the microglia population in an autocrine manner and amplified the production of inflammatory cytokines/chemokines. Furthermore, active caspase- 8 was markedly increased in the microglia in the brain tissue from patients with multiple sclerosis. Taken together, our study suggests that microglia-derived IL-1 $\beta$ via noncanonical caspase-8-dependent inflammasome is necessary for microglia to exert their pathogenic role during CNS inflammation.
\end{abstract}

\section{Introduction}

Numerous studies indicate that the inflammatory process in multiple sclerosis (MS) and experimental autoimmune encephalomyelitis (EAE) is initiated by $\mathrm{T}$ cells that are reactive against myelin (1-6). Importantly, a growing body of literature has suggested that IL-1 $\beta$ produced by the multimolecular complex known as the inflammasome is critical for the pathogenesis of a variety of CNS inflammatory processes, including MS and EAE (7-10). The NLRP3 inflammasome consists of NLRP3 linked via a homotypic pyrin domain interaction to the inflammasome adaptor molecule apoptosis-associated speck-like protein containing a C-terminal caspase-activation and recruitment (CARD) domain (ASC). The canonical NLRP3 inflammasome is activated in macrophages and dendritic cells via procaspase- 1 self-cleavage to generate active caspase-1, which processes pro-IL-1 $\beta$, resulting in release of

Authorship note: MJ and $\mathrm{HZ}$ contributed equally to this work. Conflict of interest: The authors have declared that no conflict of interest exists. License: Copyright 2018, American Society for Clinical Investigation.

Submitted: May 7, 2018; Accepted: September 18, 2018.

Reference information: / Clin Invest. 2018;128(12):5399-5412.

https://doi.org/10.1172/JCl121901. mature IL-1 $\beta$ (11-15). NLRP3 inflammasome activation in macrophages and dendritic cells is required for activation and expansion of Th17 cells during priming in the periphery (16-18). Interestingly, we found that caspase- 8 instead of caspase- 1 is critical for ASC-dependent IL-1 $\beta$ production in Th17 cells (3). Notably, we recently reported that T cell-intrinsic ASC is essential for the effector stage of EAE (3).

Microglia in the CNS continuously scrutinize their environment for damage or infection. Microglia are equipped with TLRs and P2X7R to sense TLR ligands and ATP released by dying CNS resident cells during CNS inflammatory tissue injury (19-23). We now report that microglia-intrinsic ASC is required for the effector stage of EAE, acting in the context of a noncanonical inflammasome complex. In particular, IL-1 $\beta$ is processed and produced in an ASC-NLRP3-caspase-8-dependent manner in primary microglia. Mechanistically, TLR stimulation induces IRAKM-caspase-8-ASC complex formation, resulting in the activation of caspase- 8 in the presence of ATP and IL- $1 \beta$ production in microglia.

We also found that microglia-intrinsic IRAKM-caspase-8 inflammasome activation is required for microglia survival and proliferation in the CNS of EAE mice. Consistently, active caspase- 8 was markedly increased in the microglia in brain tissue 
A

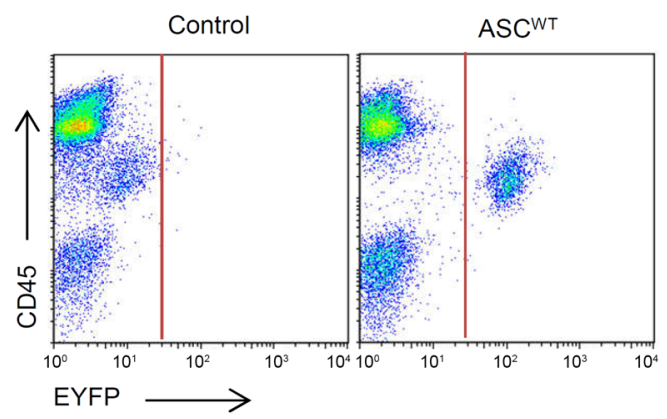

B

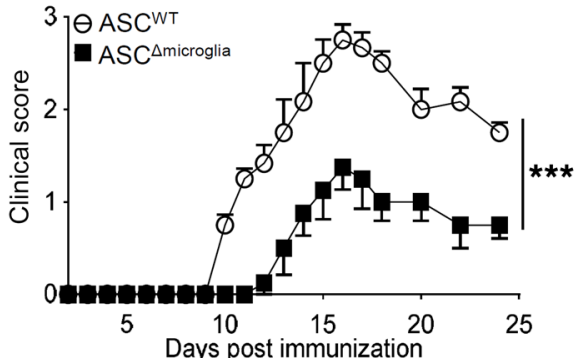

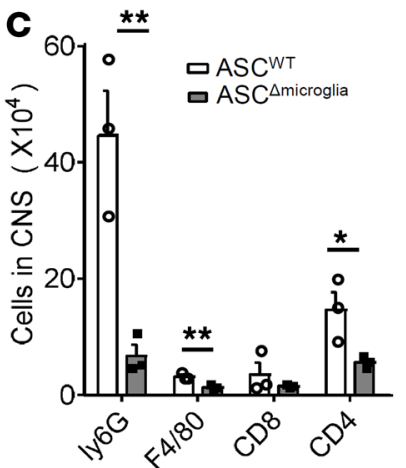

D
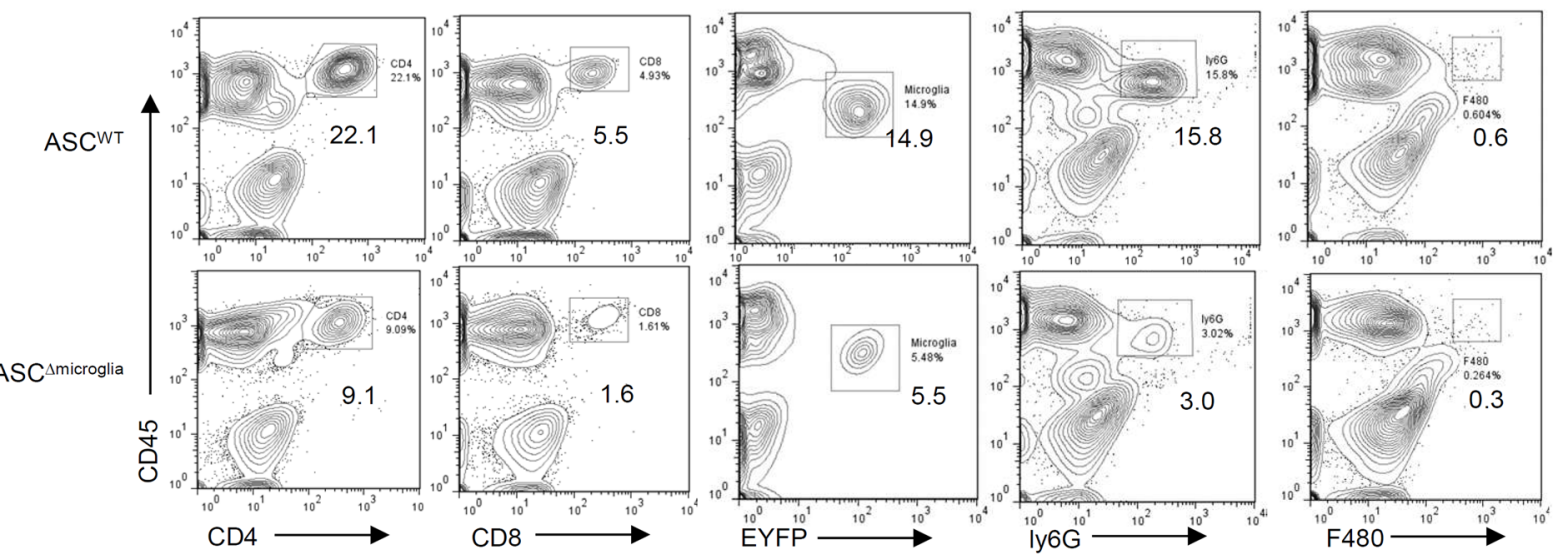

E

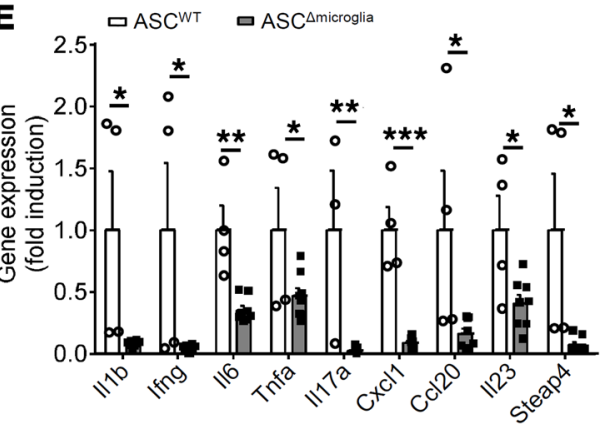

$\mathbf{F}$

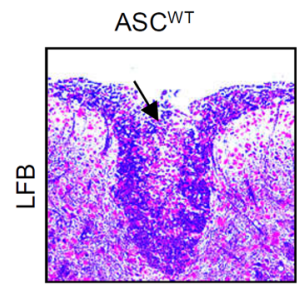

$\mathrm{ASC}^{\Delta \text { microglia }}$
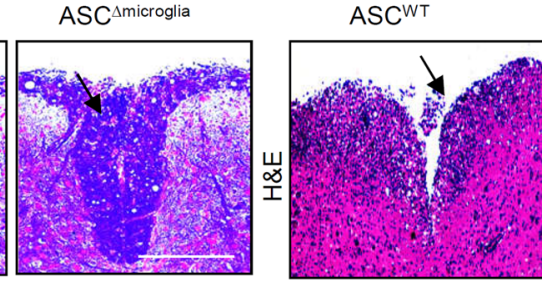

ASC $^{\Delta \text { microglia }}$
G

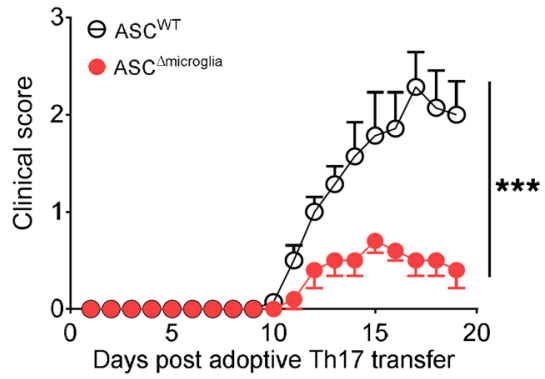

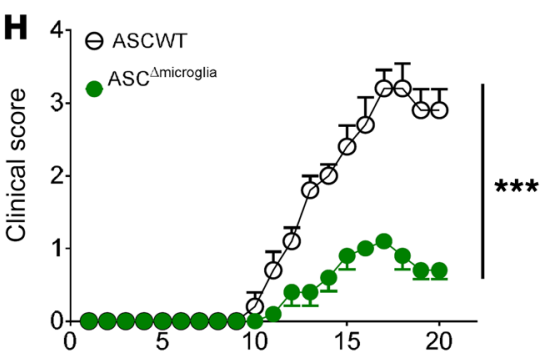

Days post adoptive Th1 transfer

Figure 1. Microglia-specific ASC deficiency attenuated EAE disease. Analysis of results for WT $\rightarrow A S C^{f l /+} C x 3 c r 7^{C r e-E R}\left(A S C^{\Delta W T}\right)$ and WT $\rightarrow A S C^{f l / f l} C \times 3 C r 7^{[r e-E R}$ (ASC ${ }^{\Delta \text { microglia) }}$ bone marrow chimera mice in EAE disease. (A) FACS analysis of CreER-EYFP expression in microglia of ASC ${ }^{\Delta W T}$ mice with or without tamoxifen administration on day 16 of EAE induced by active immunization with $\mathrm{MOG}_{35-55^{\circ}}$ (B) Mean clinical score for EAE in $\operatorname{ASC}^{\Delta W T}(n=6)$ and ASC ${ }^{\Delta m i c r o g l i a}(n=5)$ bone marrow chimera mice induced by active immunization with $\mathrm{MOG}_{35-55}$. Absolute numbers (C) and gating strategy (D) of immune cell infiltration determined at the peak of disease in brains of EAE mice by flow cytometry ( $n=3 /$ group). (E) Inflammatory gene expression in the lumbar spinal cords as assessed at the peak of disease $(n=4)$. (F) Luxol Fast Blue and H\&E staining of lumbar spinal cords harvested at the peak of disease. Scale bars: $200 \mu \mathrm{m}$. (G and $\mathbf{H})$

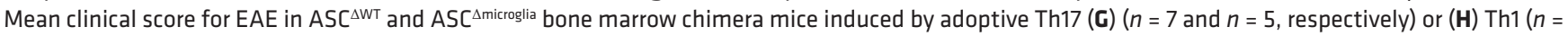
5 /group) transfer. Data are representative of 2 independent experiments; mean $\pm S E M$. ${ }^{*} P<0.05,{ }^{* *} P<0.01,{ }^{* *} P<0.001$ (unpaired 2 -tailed Student's $t$ test). EAE clinical score by 2-way ANOVA. 
from patients with MS. Notably, while IL-1R was highly induced on microglia during EAE, transfer of WT but not IL-1 $\beta$-deficient microglia was able to enhance the EAE phenotype in microgliadiminished recipient mice $\left(C x 3 c r 1^{C r e}-C s f 1 r^{f l / f l}\right.$ mice). Furthermore, $\mathrm{Ki}^{+} 7^{+}$microglia were much reduced in $\mathrm{Cx} 3 \mathrm{cr} 1^{\mathrm{Cre}}-\mathrm{Csf1} \mathrm{r}^{\mathrm{fl} / \mathrm{fl}}$ mice receiving IL-1 $\beta$-deficient microglia compared with mice transferred with WT microglia. Taken together, our results suggest that noncanonical inflammasome-derived IL-1 $\beta$ produced by microglia in the CNS helps expand the microglia population via an autocrine manner and amplifies the production of inflammatory cytokines/chemokines. As a consequence, the activated microglia are permitted to exert their pathogenic role during EAE, providing an unparalleled opportunity to develop therapeutic strategies for the treatment of MS.

\section{Results}

Microglia-intrinsic ASC is required for the effector stage of Th1- and Th17-induced EAE in the CNS. In the brain, antigen-specific $\mathrm{T}$ cells are restimulated by antigen presenting cells (APCs), leading to disease induction and progression (24-26). While dendritic cells (DCs) are professional APCs, microglia in the CNS are also potential APCs (24-26). In addition to $A s c^{f / f l} C d 11 c^{C r e}$ mice, we also bred $A s c^{f l / f l}$ mice to $C x 3 c r 1^{C r e-E R}$. To delete ASC specifically in microglia, we transferred WT bone marrow to lethally irradiated $A s c^{f l / f l} C \times 3 c r 1^{C r-E R-E Y F P}$ and control $A s c^{f l /+} C \times 3 c r 11^{C r-E R-E Y F P}$ mice, which are referred as $\mathrm{Asc}^{\Delta \mathrm{microglia}}$ and littermate control mice. Using C $x 3$ Cr1 $1^{\text {Cre-ER-EYFP }}$ bone marrow chimeric mice (transferred with WT bone marrow cells), we found that over $97 \%$ of the CD $45^{\text {lo }}$ microglia from the brain tissue gained eYFP expression, implying the high efficiency of Asc deletion in microglia of the Asc $\mathrm{smicroglia}^{-}$ mice (Figure 1A and Supplemental Figure 1A; supplemental material available online with this article; https://doi.org/10.1172/ JCI121901DS1). To further confirm these results, we also crossed Cx3cr1 ${ }^{\text {Cre-ER-EYFP }}$ mice with Rosa26-stop-DsRed to trace the efficiency of Cre recombinase in microglia. Consistently, over $95 \%$ of $\mathrm{CD} 45^{\text {lo }} \mathrm{CD} 11 \mathrm{~b}^{+}$microglia gained DsRed expression (Supplemental Figure 1B). We tested the effect of microglia-specific Asc deletion on neuroinflammation and demyelination by immunizing Asc ${ }^{\Delta \text { microglia }}$ and littermate control mice with the neuroantigen myelin oligodendrocyte glycoprotein $\left(\mathrm{MOG}_{35-55}\right)$ peptide. $\mathrm{Asc}^{\Delta \text { microglia }}$ mice had attenuated disease severity compared with controls (Figure 1B). Inflammatory mononuclear cell infiltration in the brain, including by $\mathrm{CD} 4^{+} \mathrm{T}$ cells, B cells, neutrophils, and macrophages, was similarly decreased in mice with microgliaspecific Asc ablation compared with controls (Figure 1, C and D; and Supplemental Figure 1C), and the expression of inflammatory cytokines and chemokines in the spinal cord was also significantly decreased (Figure 1E). Histopathological analysis showed decreased infiltrating immune cell accumulation and resultant demyelination in spinal cords of Asc ${ }^{\Delta \text { microglia }}$ mice compared with controls (Figure $1 \mathrm{~F}$ ). Together, these data indicate that deletion of $A S C$ from microglia protects mice from the pathogenesis of EAE, with marked attenuation of disease severity. We found that Asc deletion in microglia $\left(A s c^{\text {smicroglia }}\right)$ substantially reduced Th1- and Th17-induced EAE pathogenesis (Figure 1, G and H), with markedly reduced inflammation in the brain and spinal cord (Supplemental Figure 1, D-G).
Microglia processes IL-1 $\beta$ in an NLRP3-ASC-caspase-8-dependent manner. NLRP3 inflammasome was activated by LPS+ATP stimulation in primary microglia, as evident by pro-IL-1 $\beta$ cleavage and IL-1 $\beta$ secretion in an NLRP3/ASC-dependent manner (Figure 2, A, B, and E). Interestingly, we found that caspase-8 instead of caspase- 1 was activated in an NLRP3/ASC-dependent manner in primary microglia (Figure 2 , A and B). LPS+ATP stimulation triggered the recruitment of caspase- 8 to NLRP3-ASC in microglia (Figure 2C). Consistently, caspase-8 was recently identified as a noncanonical inflammatory caspase in myeloidlineage cells and T cells (3), and it has been shown to be capable of processing pro-IL-1 $\beta(21,27-29)$. We thus speculated that in microglia, caspase- 8 might participate in an ASC-dependent noncanonical inflammasome capable of processing pro-IL-1 13 . In response to ATP stimulation, IL-1 $\beta$ production was significantly reduced in microglia from Casp $8^{-/-}$Ripk3 $^{-/-}$mice compared with those from $\mathrm{Casp}^{+/-} \mathrm{Ripk3}^{-/-}$mice, implying a role for caspase-8 in microglia for the ASC-dependent processing of pro-IL-1 $\beta$ (Figure 2, D and E). In support of this, peptide inhibitor of caspase-8 (IETD-fmk) but not of caspase-1 (YVAD-fmk) specifically blocked IL-1 $\beta$ production in microglia (Figure $2 \mathrm{~F}$ ). Taken together, our results suggest that caspase- 8 is critical for ASC-dependent IL-1 $\beta$ production in primary microglia, which is analogue to the NLRP3-ASC-caspase-8 cascade in Th17 cells (3). This NLRP3-ASC-caspase-8 axis was operative in microglia in vivo, since FACS analysis showed caspase-8 (instead of caspase-1) was indeed activated in microglia from the brain tissue of EAE mice in an NLRP3/ASC-dependent manner (Figure 2, G and H). Importantly, endogenous TLR ligands (derived from tissue damage) could also activate this caspase-8-dependent noncanonical NLRP3 inflammasome in both human and mouse microglia (Figure 2, I and J). Additionally, genetic ablation of caspase- 8 specifically in microglia attenuated EAE, indicating the importance of this pathway for the pathogenesis of EAE (Figure $2 \mathrm{~K}$ ).

IRAKM controls the activity of caspase- 8 and $I L-1 \beta$ production in microglia. We wondered how TLR signaling leads to caspase-8 activation. Upon TLR stimulation, IRAK proteins (IRAK1-4) are recruited to the receptor complex to mediate downstream signaling pathways. By mass spectometry analysis, we found that IRAKM, but not IRAK1 or IRAK2, pulled down caspase-8 (Supplemental Figure 2). Consistently, we found that in response to LPS or LPS+ATP stimulation, caspase- 8 indeed specifically interacted with IRAKM in primary microglia (Figure 3A). IRAKM (but not IRAK1/2) formed a complex with ASC/NLRP3 in primary microglia (Figure 3, B and C). Based on these biochemical findings, we hypothesized that IRAKM may play a critical role in activating the NLRP3-ASC-caspase-8 cascade in microglia. We indeed found that IRAKM deficiency abolished LPS+ATP-induced caspase- 8 and IL-1 $\beta$ cleavage and IL-1 $\beta$ production in microglia (Figure 3, D-F). Nevertheless, LPSinduced NF- $\kappa \mathrm{B}$ activation was not affected in IRAKM-deficient microglia (Figure 3G). These results suggest that IRAKM is specifically required for the assembly of caspase-8-dependent NLRP3 inflammasome. In support of this hypothesis, we found that ASCcaspase- 8 interaction was ablated in IRAKM-deficient microglia (Figure $3 \mathrm{H}$ ), whereas IRAKM was still able to form a complex with caspase- 8 in the absence of ASC (Figure 3I). These results indicate that IRAKM first engages caspase- 8 , followed by their interaction 
A

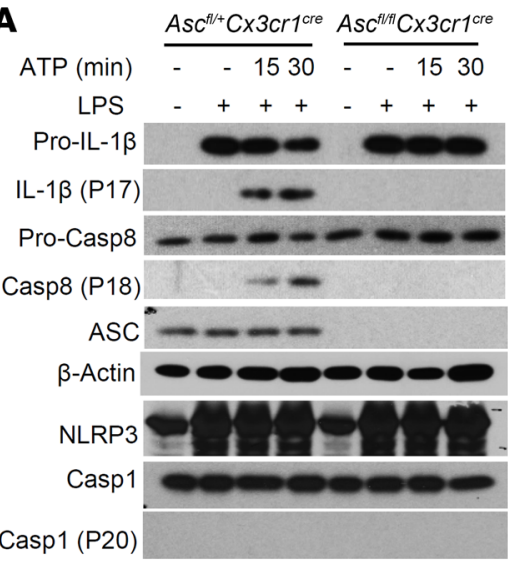

B

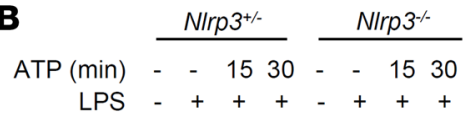

Pro-IL-1 $\beta$

IL-1ß (P17)

Pro-Casp8 $=-m-m=-$

Casp8 (P18)

ASC $-\infty=-2$

$\beta$-Actin $=00-0=0$

NLRP3
C

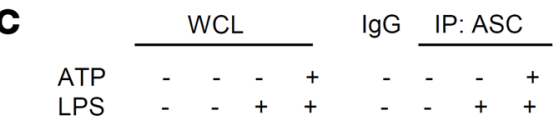

Pro-Casp8

NLRP3

ASC

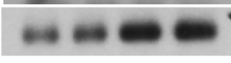

$$
\text { 玨 }
$$

Pro-Casp1

$\beta$-Actin

E

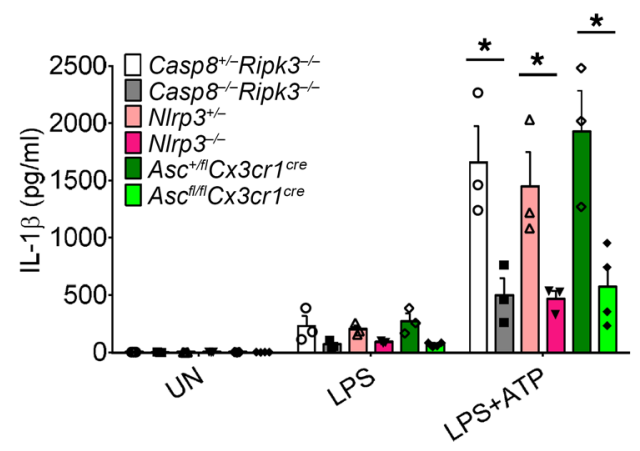

$\mathbf{F}$

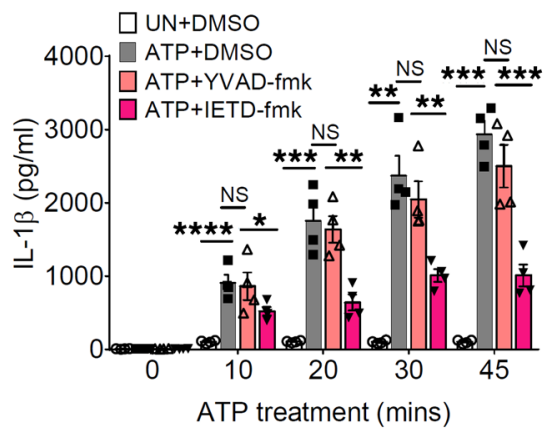

G

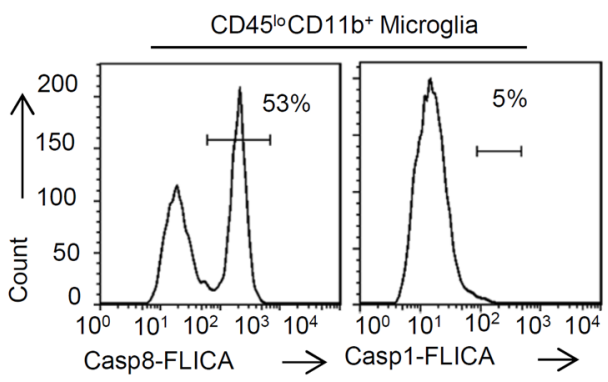

H

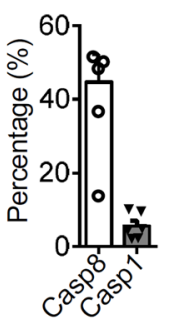

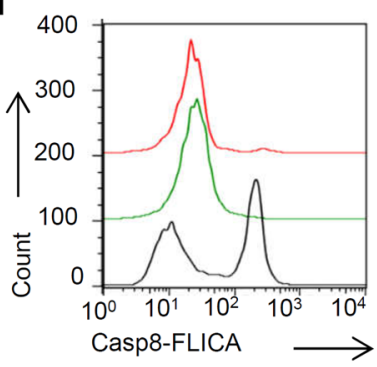

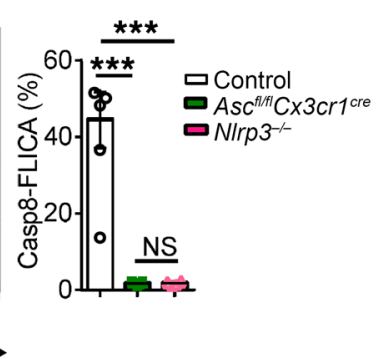

I

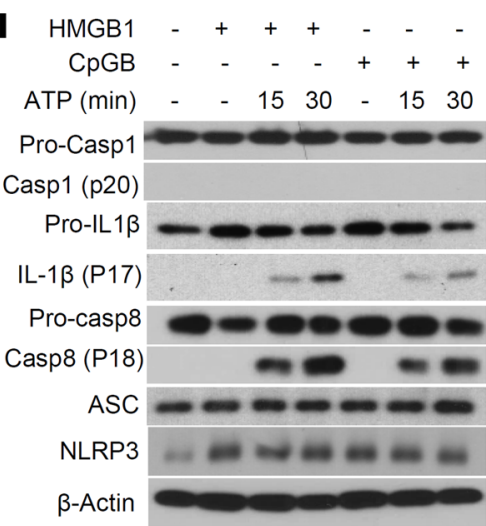

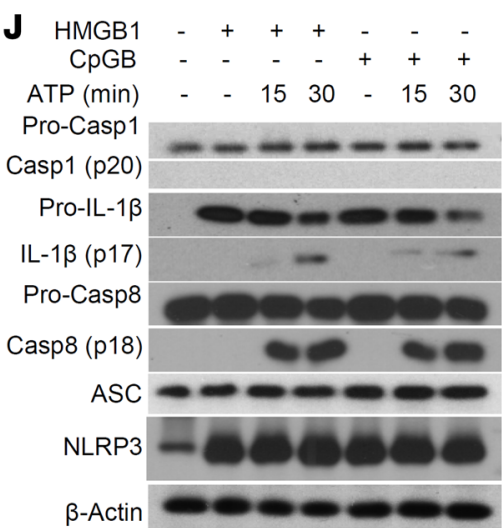

$\mathbf{K}$

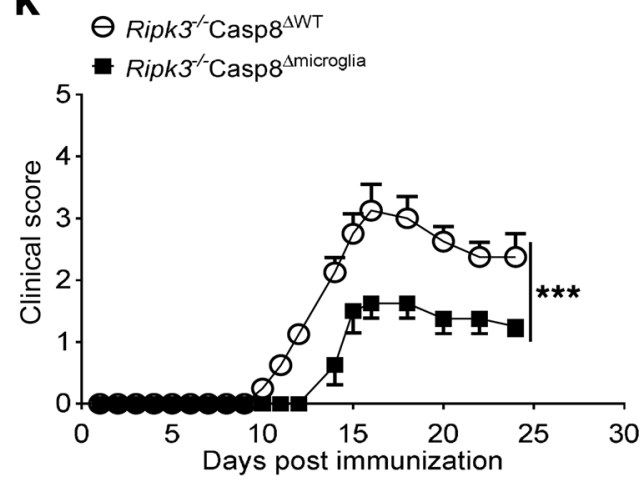


Figure 2. Microglia processes IL-1 $\beta$ in an ASC-NLRP3-caspase-8-dependent manner. (A and B) Primary microglia from 6-week-old mice were primed with LPS for 4 hours prior to stimulation with ATP for the indicated times. Cell lysate and supernatants were collected together and immunoblotted with the indicated antibodies. (C) Results of immunoblotting with antibodies to the indicated proteins for microglia stimulated with LPS (4 hours) plus ATP (30 minutes) and immunoprecipitated with anti-ASC. (D) Primary microglia from adult mice were primed with LPS for 4 hours prior to stimulation with ATP. Cell lysate and supernatants were collected together and immunoblotted with the indicated antibodies. (E) IL-1 $\beta$ ELISA of cell-free supernatants from adult mice-derived primary microglia treated with LPS for 4 hours and ATP for 15 or 30 minutes $(n=3$ / group). (F) IL-1 $\beta$ ELISA of primary microglia from adult mice treated with LPS and indicated inhibitors for 4 hours prior to stimulation with $0.2 \mathrm{mM}$ ATP for the indicated times. UN, untreated; YVAD-fmk, caspase- 1 inhibitor; IETD-fmk, caspase-8 inhibitor ( $n=4 /$ group). ( $\mathbf{G}$ and $\mathbf{H}$ ) FACS analysis of caspase-8-FLICA (fluorescent labeled inhibitors of caspases) in microglia of EAE mice at peak disease in vivo ( $n=6 /$ group). (I) Primary microglia from 6-week-old mice were stimulated with TLR agonists HMGB1 and CpCb for 8 hours prior to stimulation with $0.2 \mathrm{mM}$ ATP. Cell lysate and supernatants were collected together and immunoblotted with the indicated antibodies. (J) Human microglia cell line SV40 was stimulated with LPS $(100 \mathrm{pg} / \mathrm{ml})$ for 4 hours and ATP $(0.2 \mathrm{mM})$ for 30 or 60 minutes. Cell lysate and supernatants were collected together and immunoblotted with the indicated antibodies. (K) Clinical score for WT $\rightarrow$ Casp $8^{f / /+}[x 3 \mathrm{Cr}]^{\text {Cre-ER }}$

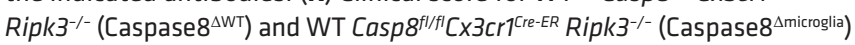
bone marrow chimera mice ( $n=6 /$ group). Data are representative of 2 independent experiments; mean $\pm \mathrm{SEM} .{ }^{*} P<0.05$, ${ }^{* *} P<0.01$, ${ }^{* *} P<$ $0.001,{ }^{* * * *} P<0.0001$ (unpaired 2-tailed Student's $t$ test). EAE clinical score by 2-way ANOVA.

with ASC for activation and cleavage of caspase-8. Interestingly, IRAKM-ASC interaction was still intact in the absence of caspase-8 (Figure 3J), indicating that IRAKM serves as a bridging molecule for ASC and caspase-8.

IRAKM is required for the activation of caspase- 8 and IL-1 $1 \beta$ production in microglia during EAE. We then examined the importance of IRAKM in caspase-8-dependent noncanonical inflammasome activation in microglia in vivo. To achieve this goal, we generated a mouse strain in which exon 3 of the gene Irak3 (which encodes IRAKM) is flanked by loxP sites (Figure 4, A and B). To delete IRAKM in microglia, we transferred WT bone marrow to lethally irradiated $\mathrm{Irakm}^{A / f l} \mathrm{C} \times 3 \mathrm{cr} 1^{\text {CreER-EYFP }}$ and control $\mathrm{Irakm}^{A /+}$ $C \times 3 c r 1^{\text {Cre-ER-EYFP }}$ mice, which are referred to as Irakm ${ }^{\text {Mmicroglia }}$ and littermate control mice. We examined the effect of microglia-specific IRAKM deletion on neuroinflammation and demyelination by immunizing $\mathrm{Irakm}^{\text {umicroglia }}$ and littermate control mice with the neuroantigen $\mathrm{MOG}_{35-55}$ peptide. Irakm ${ }^{\text {Imicroglia }}$ mice had attenuated disease severity compared with controls (Figure 4C). Inflammatory mononuclear cell infiltration in the brain, including by $\mathrm{CD}^{+} \mathrm{T}$ cells, B cells, neutrophils, and macrophages, was similarly decreased in mice with microglia-specific IRAKM ablation compared with controls (Figure 4D). Histopathological analysis showed decreased infiltrating immune cell accumulation and resultant demyelination in spinal cords of Irakm ${ }^{\text {Imicroglia }}$ mice compared with controls (Figure 4E). Together, these data indicate that deletion of IRAKM from microglia protects mice from the pathogenesis of EAE, with marked attenuation of disease severity. Consistent with the ex vivo data, caspase-8 activation and IL-1 $\beta$ production in primary microglia from the EAE mice were substantially reduced by IRAKM deficiency (Figure 4, F and G).
Microglia-intrinsic caspase-8 inflammasome activation is required for microglia survival and proliferation in the CNS. We next investigated how caspase-8-dependent noncanonical inflammasome activation in microglia contributes to EAE pathogenesis. Caspase- 8 was indeed activated in the expanded microglia in the CNS during the course of EAE (Figure 5, A and B). Importantly, active caspase- 8 was increased in the microglia in brain tissue from patients with MS (Figure 5, C and D, and Supplemental Table 1). Furthermore, we detected IL-1 $\beta$ production in sorted microglia from brain tissue of EAE mice (Figure 5E). Interestingly, we found IL-1R was highly induced on microglia from brain tissue of EAE mice (Figure 5F). In the brain tissue of EAE mice, we noted that $46 \%$ of the IL- $1 \mathrm{R}^{+}$caspase $-8^{+}$microglia were $\mathrm{Ki} 67^{+}$, whereas only $18 \%$ of IL-1R $\mathrm{R}^{-}$caspase- $8^{-}$microglia were $\mathrm{Ki}^{+} \mathrm{7}^{+}$, suggesting that caspase-8-dependent IL-1 $\beta$ production by microglia in the CNS might directly act on microglia in an autocrine manner (Figure $6 \mathrm{~A}$ ). In support of an autocrine action of IL- $1 \beta$ in this ex vivo system, we found that IL-1RA treatment also reduced cell survival of LPS+ATP-treated microglia (Figure 6B). The removal of the IRAKM-caspase-8-ASC-NLRP3 axis increased cell death in LPS+ATP-treated microglia (Figure 6B). While caspase- $8^{+}$microglia as well as $\mathrm{Ki}^{-} 7^{+}$and $\mathrm{Brdu}{ }^{+}$microglia were substantially reduced in the spinal cord tissues of EAE mice with microglia-specific deletion of ASC, IRAKM, or caspase-8 (Figure 6, C and D), establishing the IRAKM-caspase-8-ASC axis in promoting microglia survival and proliferation contributed to EAE pathogenesis. Flow cytometry analysis of the infiltrated cells in brain tissues indicated that the microglia have enhanced cell proliferation (Ki67 $7^{+}$cells) in the WT mice as compared with those in microglia-specific ASC-, IRAKM-, or caspase-8-deficient mice (Figure 6E). It was reported that specific deletion of CSF1R in microglia $\left(\mathrm{Cs} f 1 r^{\mathrm{f} / \mathrm{f}} \mathrm{C} \times 3 \mathrm{cr} 1^{\mathrm{Crr}}\right)$ diminishes the microglia population in the CNS (Supplemental Figure 3A). We transferred WT and IL-1 $\beta$-deficient microglia into the brains of $C s f 1 r^{f / f} \mathrm{C} \times 3 \mathrm{crr}^{\mathrm{Cre}}$ mice after tamoxifen-induced deletion of CSF1R in microglia (Supplemental Figure 3, A and B). We found that WT but not IL-1 $\beta$-deficient microglia were able to enhance the EAE phenotype, indicating the pathogenic role of microgliaderived IL-1 $\beta$ in EAE (Figure 6F). Consistently, we showed that Csfl $1 r^{\nexists / f} \mathrm{C} \times 3 \mathrm{cr} 1^{\mathrm{Cre}}$ mice (treated with tamoxifen) without microglia transfer displayed reduced EAE disease compared with mice transferred with WT microglia (Supplemental Figure 3C), further supporting the pathogenic role of microglia-derived IL-1 $\beta$ in EAE. Furthermore, the microglia numbers were reduced in the brains of mice transferred with IL-1 $\beta$-deficient microglia compared with mice that received WT microglia (Figure $6 \mathrm{G}$ ), supporting the impact of microglia-derived IL-1 $\beta$ on microglia survival during EAE. Moreover, $\mathrm{Ki}^{+} 7^{+}$and Brdu ${ }^{+}$microglia were much reduced in $C \times 3 c r 1^{C r e}-C s f 1 r^{f / f / l}$ mice receiving IL-1 $\beta$-deficient microglia compared with mice that were transferred with WT microglia (Figure $6, \mathrm{H}-\mathrm{J})$. Compared with WT microglia, $\mathrm{Il}_{1} b^{-/-}$microglia from EAE brains produce less inflammatory cytokines and chemokines, indicating IL-1 $\beta$ signaling is critical for promoting microglia activation (Supplemental Figure 3D).

Microglia-intrinsic IRAKM-caspase-8 inflammasome activation is required for neutrophil recruitment in the CNS during EAE. We next investigated the consequence of IRAKM-caspase-8-dependent microglia survival/proliferation on EAE pathogenesis. In 
A

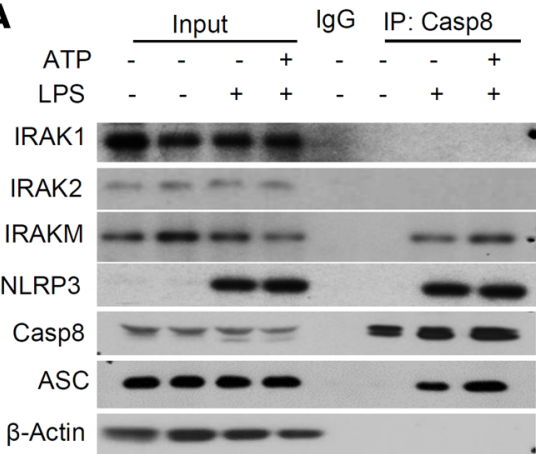

D
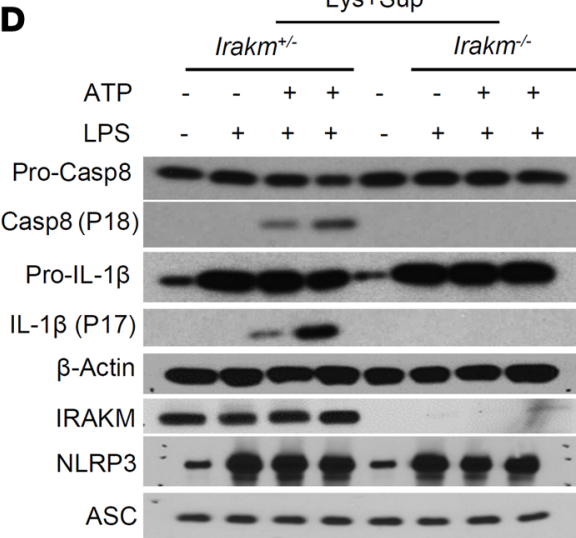

G
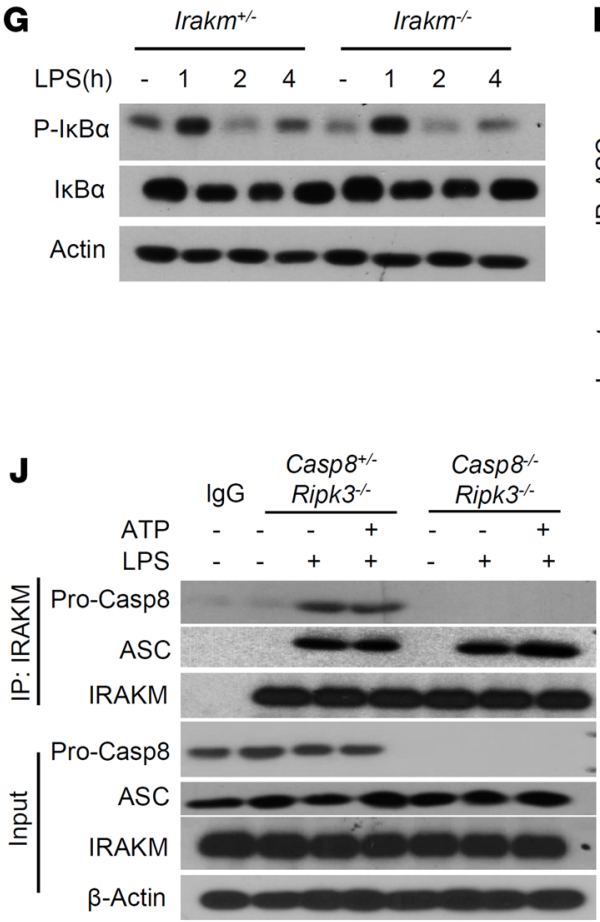

B

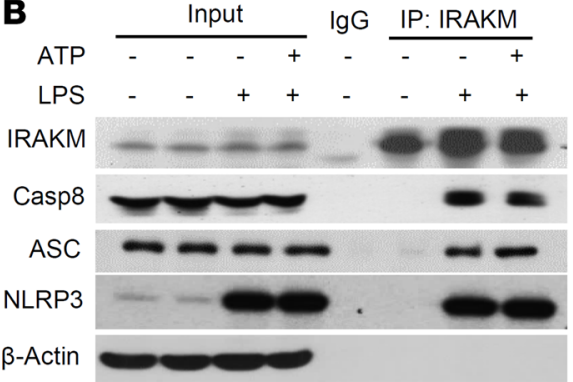

$\mathbf{E}$
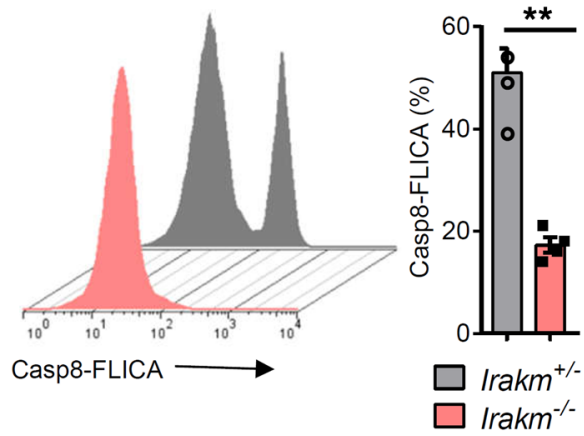

C
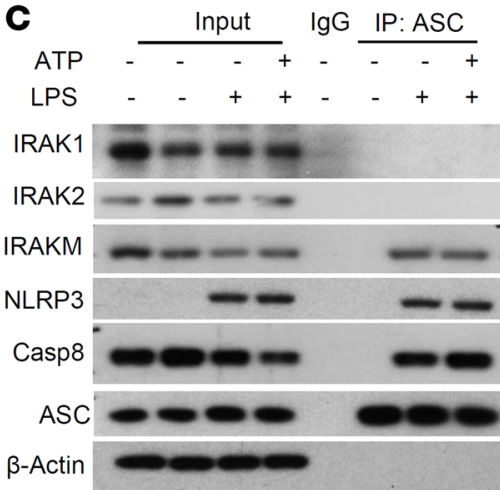

$\mathbf{F}$

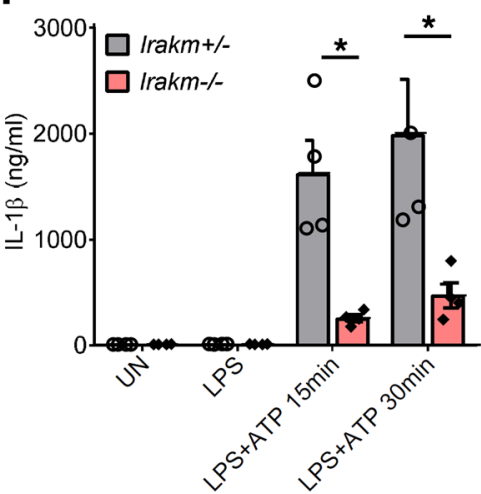

H

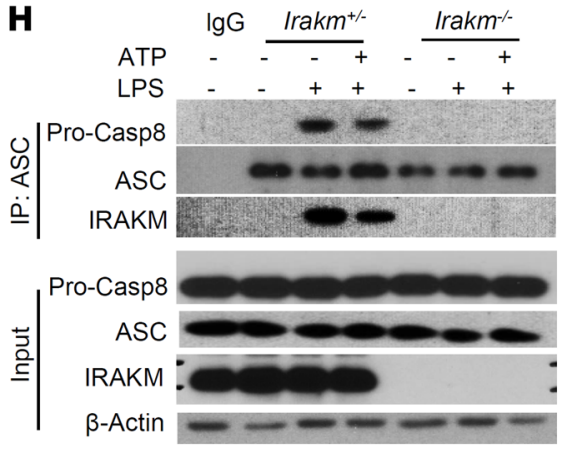

I

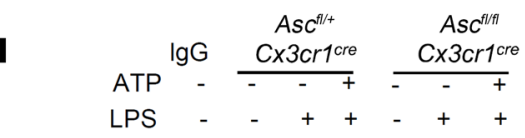

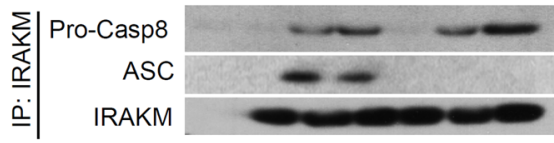

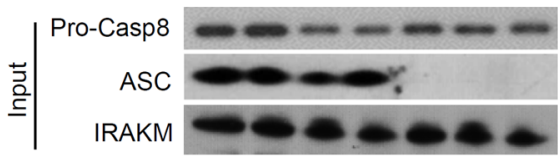

Figure 3. IRAKM controls the activity of caspase- 8 and IL-1 $\beta$ production in microglia. (A-C) Results of immunoblotting with antibodies to the indicated proteins for primary microglia stimulated with LPS (4 hours) plus ATP (30 minutes) and immunoprecipitated with anti-caspase-8 (A), anti-IRAKM (B), or anti-ASC (C). (D) Primary microglia from 6-week-old mice with indicated genotyping were primed with LPS $(100 \mathrm{pg} / \mathrm{ml})$ for 4 hours prior to stimulation with $0.5 \mathrm{mM}$ ATP for the indicated times. Cell lysate and supernatants were collected together and immunoblotted with the indicated antibodies. (E) Primary microglia from 6-week-old mice with indicated genotypes was stimulated with LPS ( $0.1 \mu \mathrm{g} / \mathrm{ml}, 4$ hours) plus ATP (30 minutes) and stained with caspase-8-FLICA in the last hour, followed by flow analysis of caspase-8 activation ( $n=4$ /group). (F) IL-1 $\beta$ ELISA of cell-free supernatants from adult mice-derived primary microglia treated with LPS $(100 \mathrm{pg} / \mathrm{ml})$ for 4 hours and ATP $(0.2 \mathrm{mM})$ for 15 or 30 minutes ( $n=4 /$ group). (C) Primary microglia from mice with indicated genotypes was primed with LPS $(100 \mathrm{pg} / \mathrm{ml})$ for 4 hours prior to stimulation with $0.2 \mathrm{mM}$ ATP for the indicated times. Cell lysate and supernatants were collected together and immunoblotted with the indicated antibodies. (H-J) Results of immunoblotting with antibodies to the indicated proteins for primary microglia stimulated with LPS (100 $\mathrm{pg} / \mathrm{ml}, 4$ hours) plus ATP (30 minutes) and immunoprecipitated with anti-ASC (H) or anti-IRAKM (I and J). Data are representative of 2 independent experiments; mean \pm SEM. ${ }^{*} P<0.05$ (unpaired 2-tailed Student's $t$ test). 
A

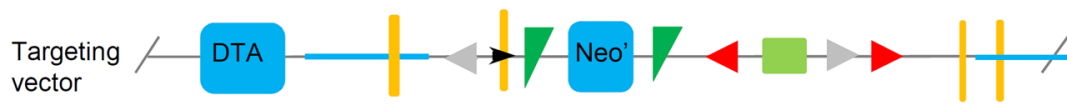

After Cre recombination
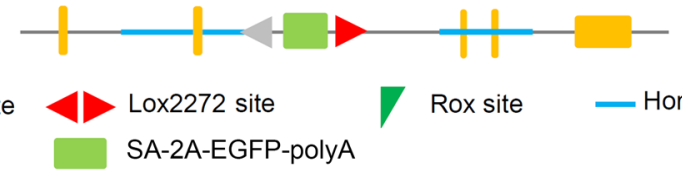

Loxp site
Exon

Lox2272 site

SA-2A-EGFP-polyA
B

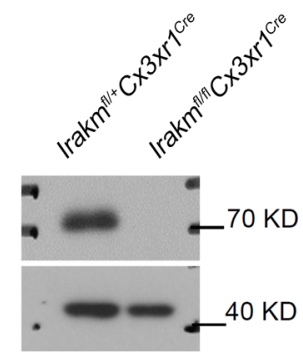

C

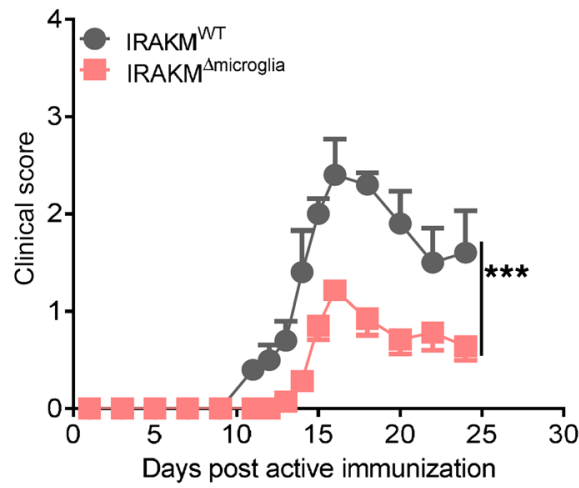

D

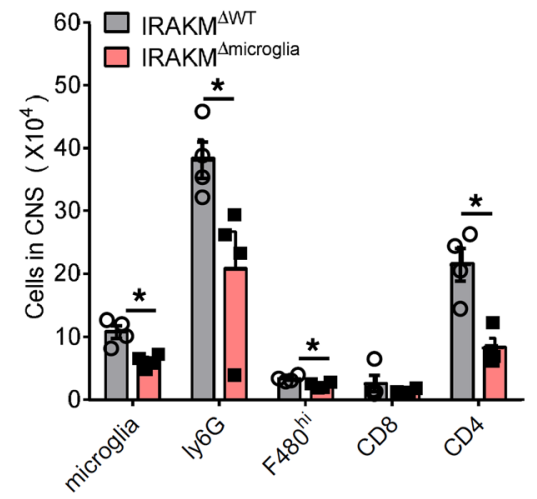

E

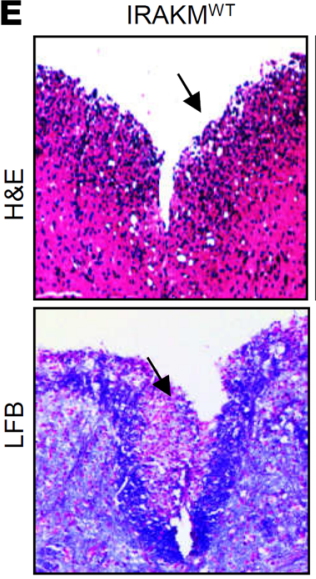

IRAKM $^{\Delta \text { microglia }}$

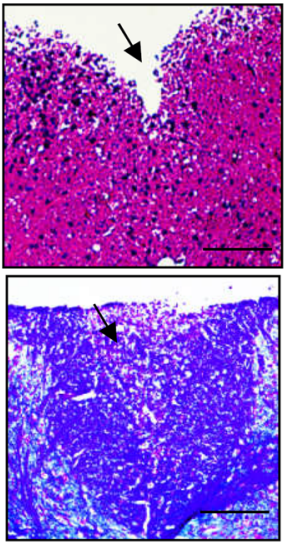

$\mathbf{F}$

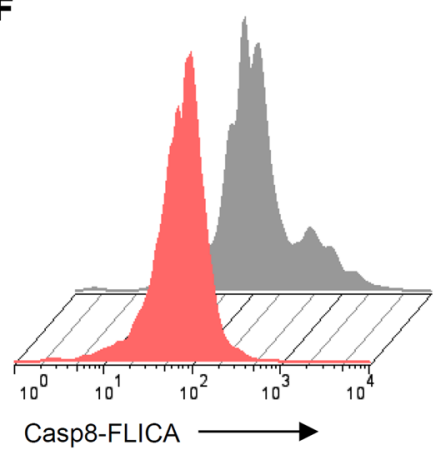

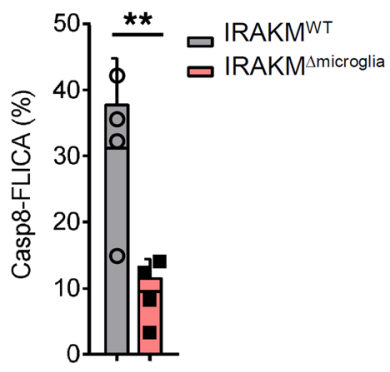

G

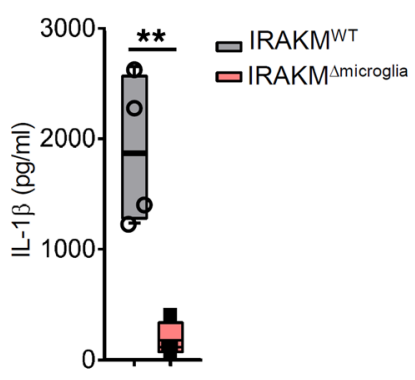

Figure 4. IRAKM controls the activity of caspase-8 and IL-1 $\beta$ production in microglia in vivo. (A and $\mathbf{B})$ Targeting vector design for generation of a mouse strain with Irak3 exon 3 flanked by loxp sites (A), and Western blot analysis of IRAKM expression in FACS-sorted microglia from indicated mice (B). (C)

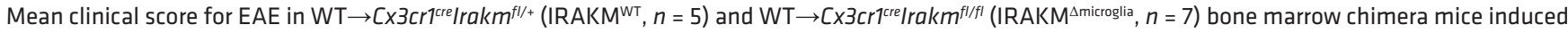
by active immunization with $\mathrm{MOG}_{35-55^{\circ}}$ (D) Absolute numbers of immune cell infiltration as well as resident microglia determined at the peak of disease in brains of EAE mice by flow cytometry ( $n=3$ /group). (E) H\&E and Luxol Fast Blue staining of lumbar spinal cords harvested at the peak of disease. Scale bars: $200 \mu \mathrm{m}$. (F) Flow cytometry analysis of caspase-8 activation in microglia of EAE mice at peak disease $(n=4)$. (G) Primary microglia of EAE brains were isolated and cultured for 24 hours ex vivo. Cell-free supernatant was collected for IL-1 $\beta$ ELISA $(n=4)$. Data are representative of 2 independent experiments; mean \pm SEM. ${ }^{*} P<0.05,{ }^{* *} P<0.01$ (unpaired 2-tailed Student's $t$ test). EAE clinical score by 2 -way ANOVA.

addition to IL-1 $\beta$ production, we also detected release of other cytokines and chemokines from sorted microglia from brain tissue of EAE mice (Figure 7A), including IL-23, IL-6, CXCL-1, CXCL2, and CXCL5. Notably, microglia expansion was readily datable at day 10, peaking at day 16 (peak of disease) upon immunization (Figure 7B). Interestingly, both IL-17- and IFN- $\gamma$-producing cells were reduced at day 10 and day 16 (the peak of the disease) in the brain tissue of microglia-specific ASC- or IRAKM-deficient mice compared with that of WT mice (Figure 7C). Likewise, microgliaspecific deficiency in ASC or IRAKM also had a substantial impact on the neutrophils in the brain tissue as early as day 10 upon immunization (Figure 7D), which coincided with the microglia activation and expansion in the WT cells (Figure 7A). Consistently, IL-17/IFN- $\gamma$-producing cells and neutrophils were reduced in the brain tissue of mice transferred with IL-1 $1 \beta$-deficient microglia compared with mice that received WT microglia (Figure 7E).

\section{Discussion}

Microglia have been shown to sense TLR ligands and ATP released by dying CNS resident cells during neuroinflammation (19-23). This study reports a mechanism for noncanonical NLRP3 inflammasome activation in adult microglia for the effector stage of EAE. Our data demonstrated that TLR stimulation leads to IRAKM-caspase-8-ASC complex formation, resulting in the acti- 
A

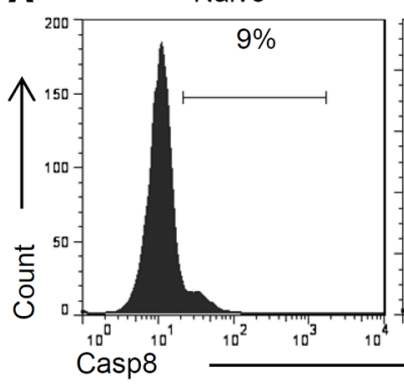

C

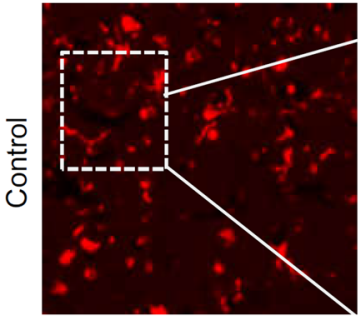

TMEM119/Casp8-FLICA
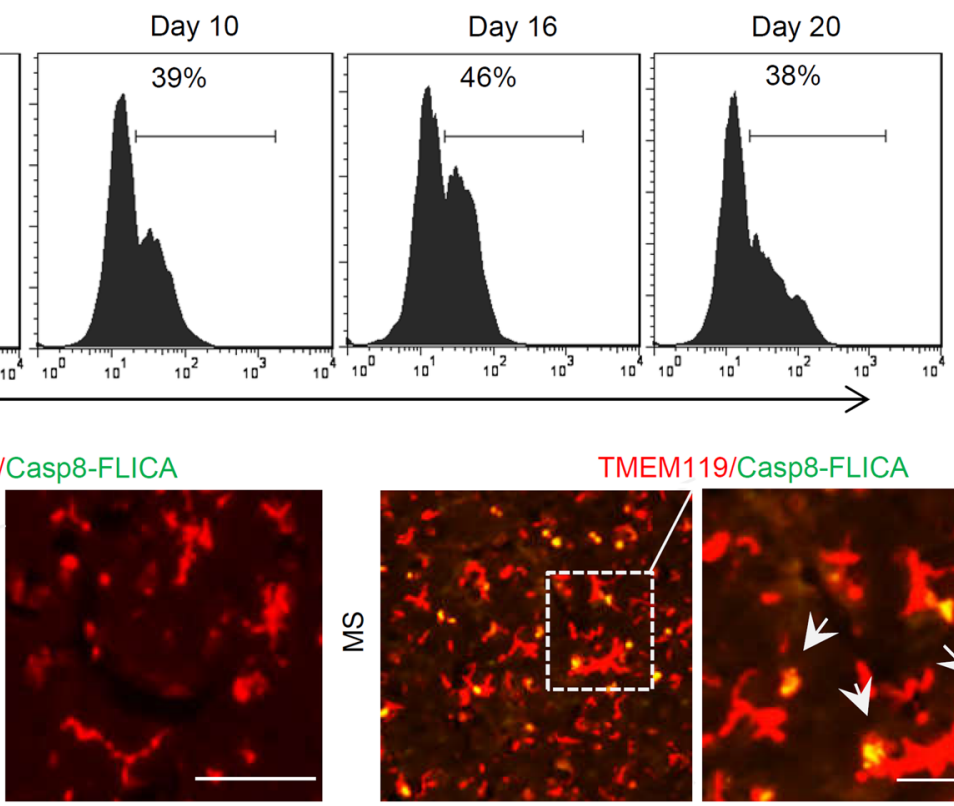

Day 10

$\mathbf{E}$

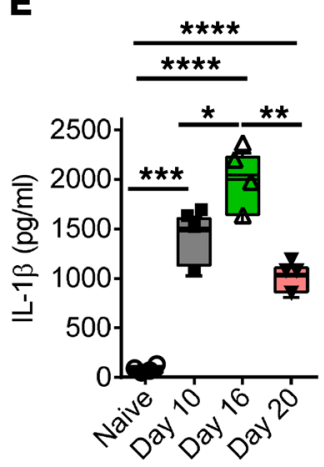

$\mathbf{F}$

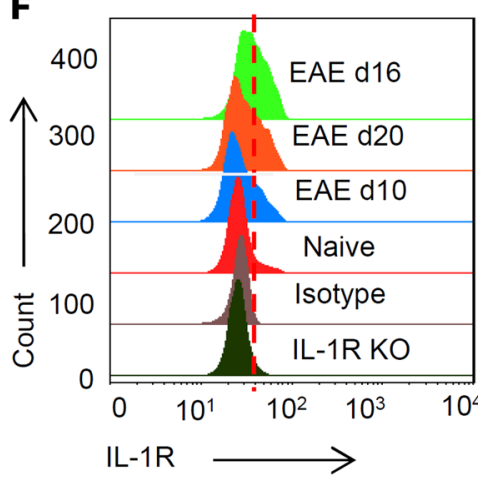

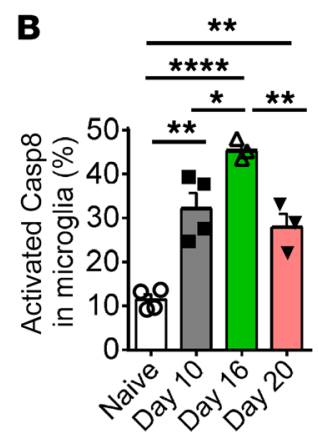

D
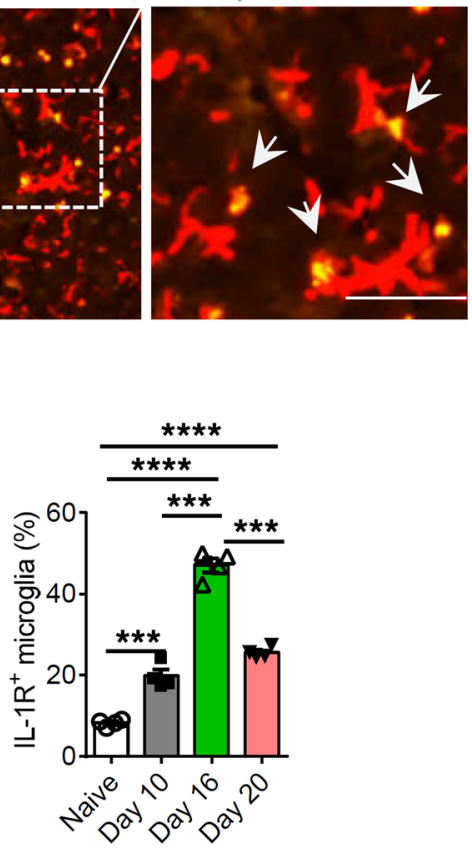

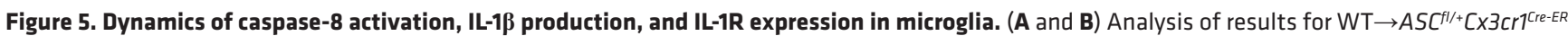
$\left(A S C^{\triangle W T}\right.$ ) bone marrow chimeric mice in EAE disease. Flow cytometry analysis of activated caspase-8 in primary EYFP+ microglia isolated from brains of EAE mice at the indicated time points $(n=4)$. The quantification of activated caspase ${ }^{+}$microglia is shown in $\mathbf{B}$. (C and $\left.\mathbf{D}\right)$ Brain specimens of 7 controls and 11 MS cases were provided by the Multiple Sclerosis Tissue Bank at Imperial College London. The edges of cortical active lesions in brain slices were stained with the indicated antibodies against microglia markers TMEM119 and activated caspase-8-FLICA. Scale bars: $50 \mu \mathrm{m}$ (C). The activated caspase-8 ${ }^{+}$ cells were quantified (D). (E) EFYP+ primary microglia sorted from EAE mice (ASC ${ }^{\Delta W T}$ ) at the indicated times was cultured overnight and supernatant was subjected to ELISA analysis of IL-1 $\beta$ production $(n=4)$. (F) FACS analysis of IL-1R expression in EFYP' primary microglia isolated from brains of WT naive and EAE mice (ASC $\left.{ }^{\triangle W T}\right)(n=4)$. Data are representative of 2 independent experiments; mean \pm SEM. ${ }^{*} P<0.05,{ }^{* *} P<0.01,{ }^{* * *} P<0.001,{ }^{* * *} P<0.0001$ (unpaired 2-tailed Student's $t$ test).

vation of caspase- 8 and IL-1 $1 \beta$ release in microglia. Notably, active caspase- 8 was also markedly elevated in microglia in the brain tissue from patients with MS. We found that IL-1 $\beta$ produced by microglia in the CNS facilitates the expansion of the microglia population in an autocrine manner, resulting in the production of inflammatory cytokines/chemokines. Consequently, microglia-specific ASC deficiency attenuated T cell expansion and neutrophil recruitment during EAE pathogenesis. This study helps to define a molecular mechanism responsible for the noncanonical inflammasome activation in a critical cell type of EAE pathogenesis, providing an unparalleled opportunity to develop therapeutic strategies for the treatment of MS.

While the microglial compartment is quite stable during homeostasis, microglial proliferation and expansion are detected during CNS pathology $(30,31)$. Ajami et al. reported microglia proliferation in the spinal cords of EAE mice (30). However, due to the design of the study, they were unable to completely exclude the possible contribution of infiltrated monocytes to the $\mathrm{BrdU}^{+}$ Iba- $1^{+}$cells in the spinal cords of EAE mice. More recently, Bruttger et al. showed microglia repopulated in response to depletion by diphtheria toxin was exclusively derived from CNS-resident cells, without contribution from bone marrow-derived cells (32). Furthermore, inhibition of IL-1R signaling abolished microglia repopulation whereas high IL-1R expression was detected on proliferating microglia in the microglia depletion model (32), suggesting the critical role of IL-1 signaling in driving microglia survival/ proliferation. However, the mechanism by which microglial proliferation is regulated in pathophysiological context has not been 
A

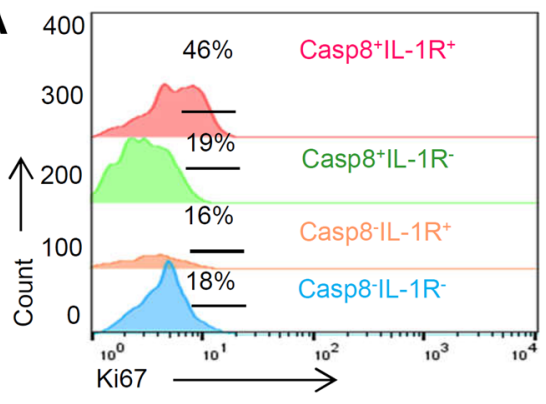

C
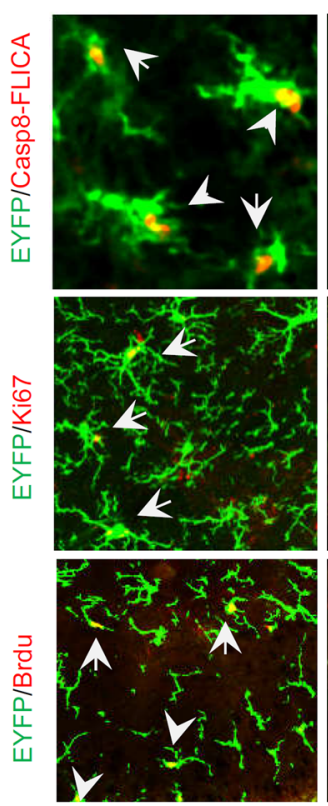

E

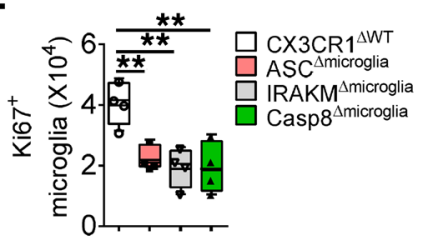

】

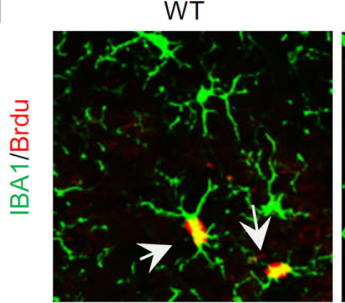

$\mathrm{ASC}^{\Delta \text { microglia }}$
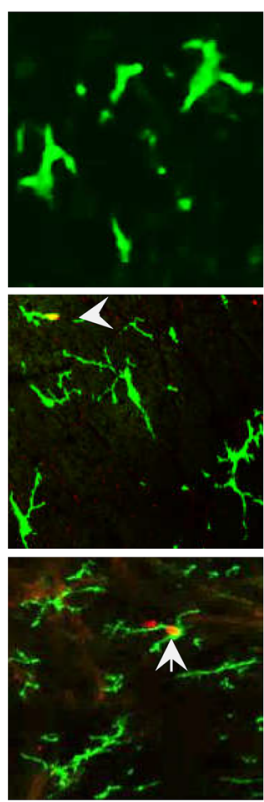

$\mathbf{F}$

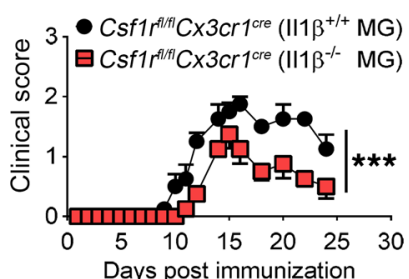

$\operatorname{IRAKM}^{\Delta \text { microglia }}$
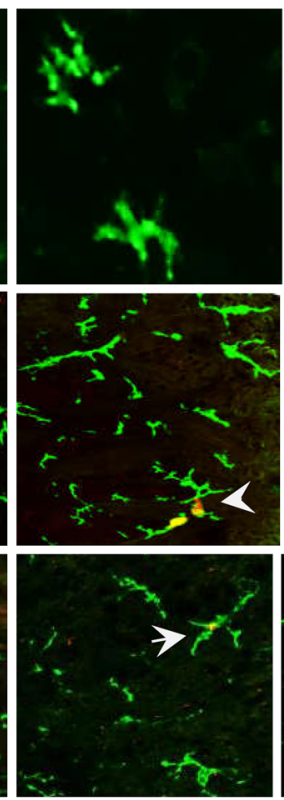
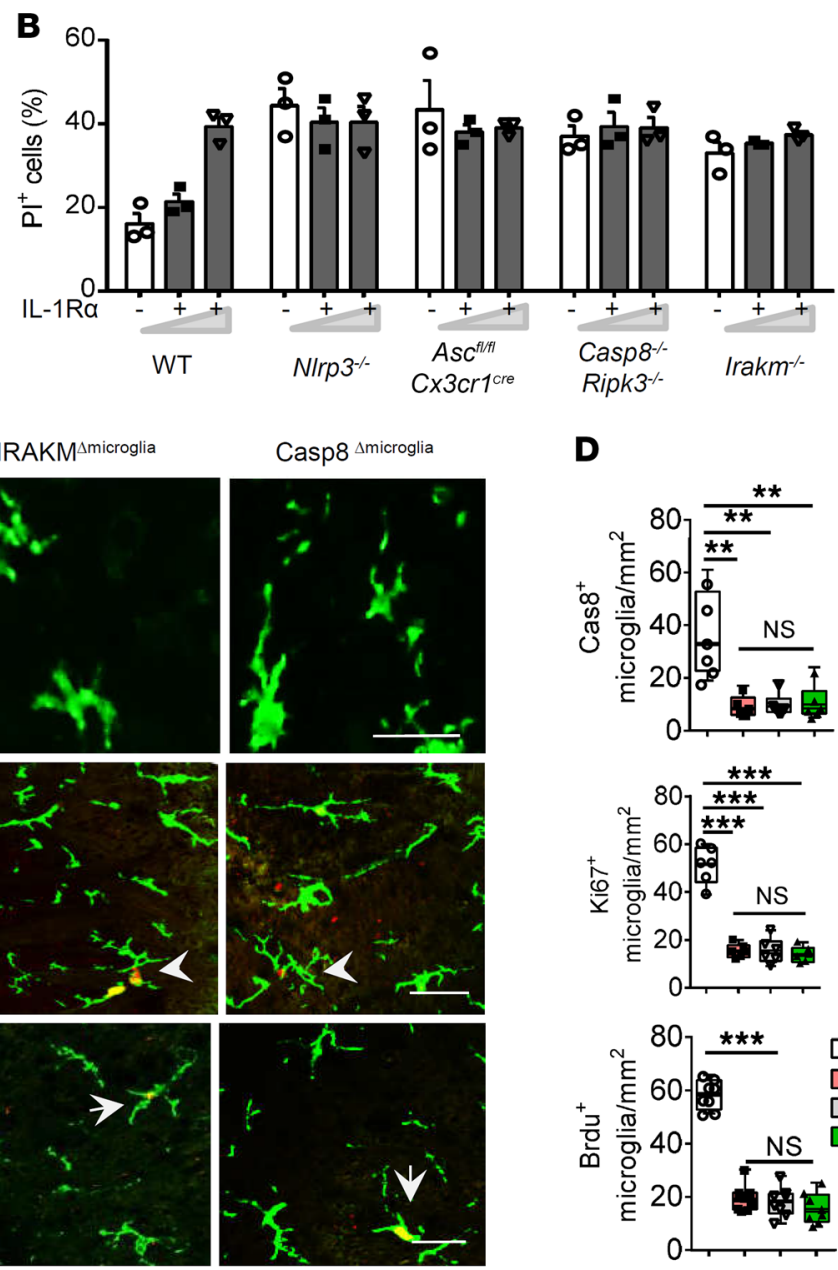

G

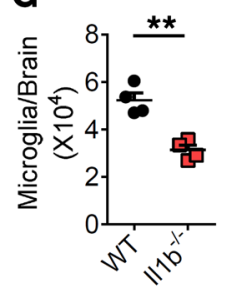

D
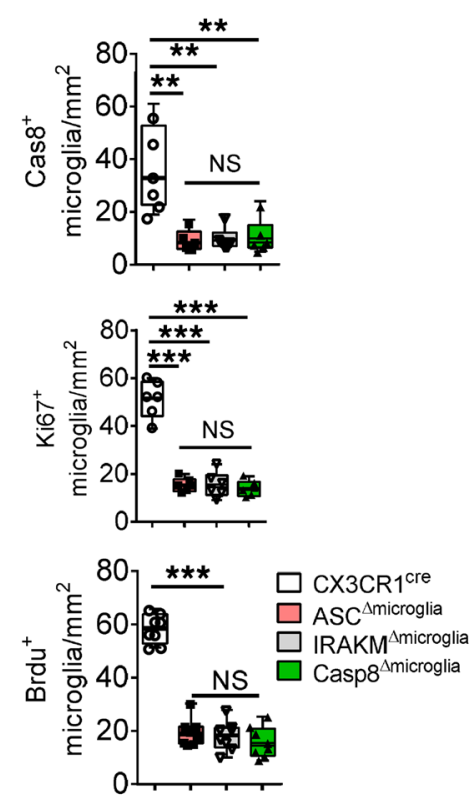

H

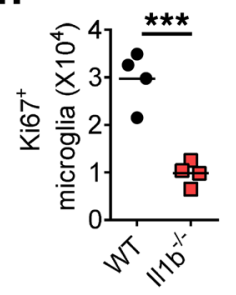

Figure 6. Microglia-intrinsic caspase-8 inflammasome activation is required for microglia survival and proliferation in the CNS. (A) The proliferation of microglia from WT EAE mouse brain at peak disease was analyzed by flow cytometry after staining with antibodies against CD45, CD11b, IL-1R, Ki67, and caspase-8-FLICA. (B) Primary microglia isolated from mouse brain with indicated genotypes were stimulated with LPS+ATP in the presence of IL-1R $\alpha$ followed by PI staining and flow cytometry analysis $(n=3)$. (C and $\mathbf{D})$ Spinal cord from EAE mice with indicated genotypes at peak disease were stained with antibodies against caspase-8-FLICA, Ki67, and Brdu. EYFP indicates the microglia ( $n=6 /$ group). WT $\rightarrow\left[\times 3 c r 7^{\text {Cre-ER }}\right.$ (CX3Cr1 ${ }^{\mathrm{AWT}}$ ). Scale bars, $50 \mu \mathrm{mm}$. (E) Flow cytometry analysis of Ki67 microglia in brains of EAE mice with indicated genotypes $(n=6)$. (F-J) WT and II1 $b^{-/-}$microglia isolated from adult mouse brain were transferred to $\left[s f 1 r^{f l / f l} C \times 3 c r 1^{\text {cre }}\right.$ mice after the fourth tamoxifen injection $(5 \mathrm{mg} /$ mouse/week, i.p.) and the mice were subjected to EAE induction 2 weeks after the last tamoxifen injection $(n=6)$. Clinical score was presented as mean \pm SEM $(\mathbf{F})$. Total microglia $(\mathbf{C})$ and Ki67+ microglia $(\mathbf{H})$ in brains at EAE peak were analyzed by flow cytometry. Brdu+ microglia were analyzed in brain slices (I and J). Scale bar, $50 \mu \mathrm{m}$. Data are representative of 2 independent experiments; mean \pm SEM. ${ }^{*} P<0.05,{ }^{* *} P<0.01,{ }^{* * *} P<0.001$ (unpaired 2-tailed Student's $t$ test). EAE clinical score by 2 -way ANOVA. 

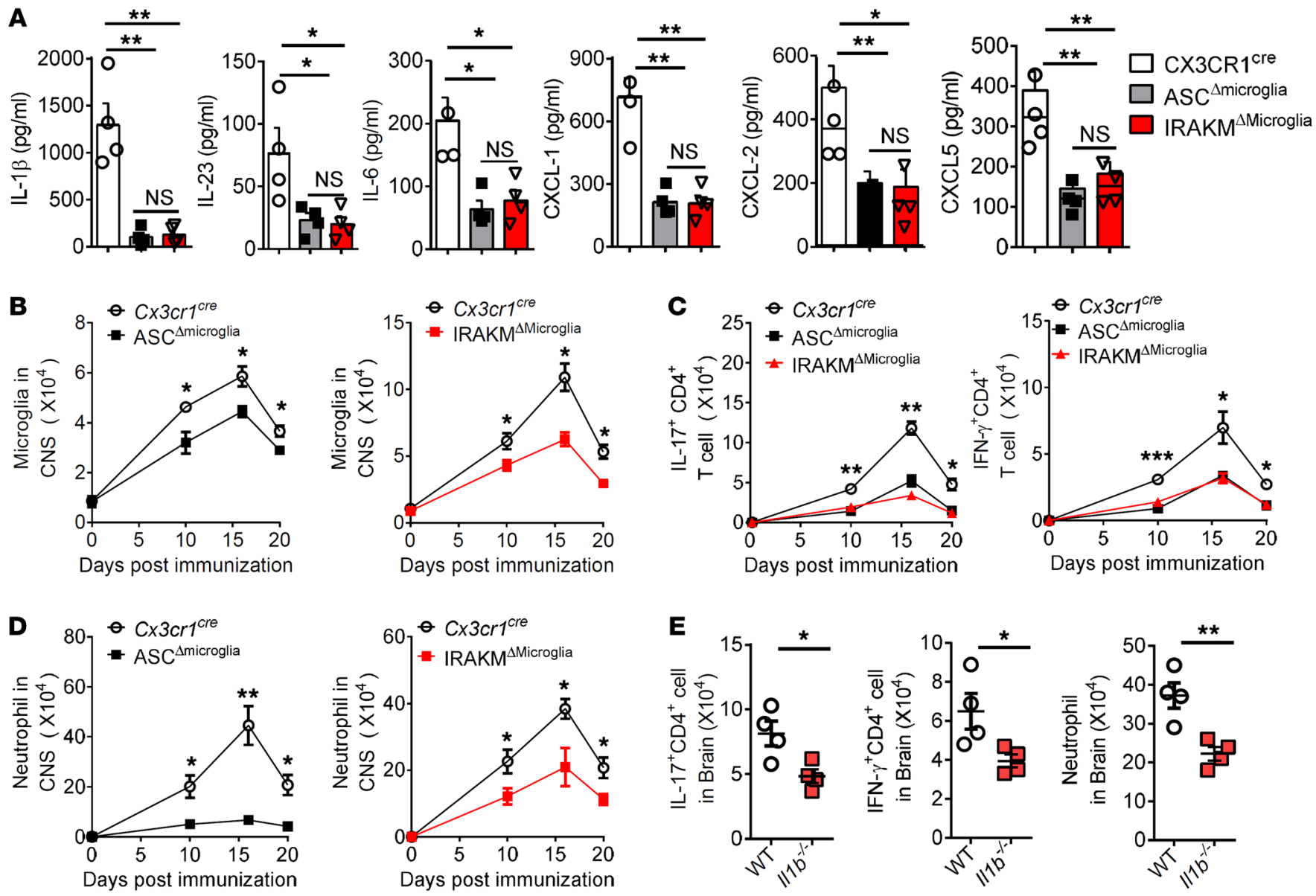

Figure 7. Microglia-intrinsic IRAKM-caspase-8 inflammasome activation is required for neutrophil recruitment and T cell expansion in the CNS. (A) Microglia were isolated from brains of EAE mice with indicated genotypes at peak disease and cultured for 24 hours. The supernatant was analyzed by ELISA for cytokine and chemokine production. (B-D) Flow cytometry analysis of microglia (B), IL-17+CD4+, IFN- $\gamma^{+}$CD4+ cell (C) and neutrophil (D) in brains of EAE

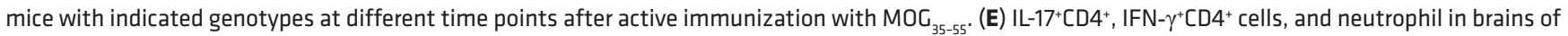
mice indicated in Figure $6 \mathrm{~F}$ were analyzed by flow cytometry. Data are representative of 2 independent experiments $(n=4 /$ group $)$. Mean \pm SEM. ${ }^{*} P<0.05$, ${ }^{* *} P<0.01$ (unpaired 2-tailed Student's $t$ test).

established. Our current work extends the previous studies and represents the first demonstration that IL-1 $\beta$ affects microglial proliferation during the pathogenesis of EAE.

The study by Ofengaim et al. reported the activation of necroptosis in multiple sclerosis (33). It is well-documented in the literature that caspase- 8 has to be inhibited for necroptosis to take place. Compared with those of age-matched controls, tissue samples from cortical lesions of the patients with MS showed a reduction in the levels of active caspase-8. After induction of EAE, the spinal cord level of the active caspase- 8 subunit was decreased. While total tissue lysates were used in assessing the levels of active caspase- 8 by Western blot analysis, Ofengaim et al. showed that activation of necroptosis was mostly detected in oligodendrocytes in $\mathrm{MS}\left(\mathrm{CC}^{+} \mathrm{MLKL}^{+}\right.$cells) and EAE lesions $\left(\mathrm{MBP}^{+} \mathrm{RIPK} 1^{+}\right.$cells) (33). Interestingly, we detected more active caspase- 8 in microglia of MS lesions compared with those of normal tissue. Future studies are required to investigate the mechanism and consequence of differential caspase-8 activation in various CNS resident cells during the pathogenesis of EAE and MS.
Notably, we previously reported that caspase-8 instead of caspase- 1 is also critical for ASC-dependent IL-1 $\beta$ production in Th17 cells (3). T cell antigen receptor (TCR) activation induced pro-IL-1 $\beta$ expression and nuclear-to-cytosolic translocation of ASC; polarized Th17 cells expressed IL-1R (3), and they produced mature IL-1 $\beta$ in response to ATP via ASC- and NLRP3-dependent caspase-8 activation. While IRAKM is a signaling molecule under the TLR-mediated pathway, IRAKM expression was not detected in T cells (our unpublished data). Consistently, IRAKM deficiency did not have any impact on ASC-dependent IL-1 $\beta$ production in Th17 cells (our unpublished data). Thus, IRAKM-mediated ASCcaspase- 8 activation and IL-1 $1 \beta$ production represents a signaling pathway unique to microglia (Figure 8). In support of our study, it was previously reported that caspase- 8 activation in microglia leads to IL-1 $\beta$ production without causing cell death, and depletion or inhibition of caspase-8 attenuated microglia activation accompanied by reduced neurotoxicity $(21,34)$.

Upon MOG immunization, IL-1R expression was detected in $\mathrm{IL}-17^{+} \mathrm{CD} 4^{+} \mathrm{T}$ cells, but not in IFN $-\gamma^{+} \mathrm{CD} 4^{+} \mathrm{T}$ cells (3). Th17 cells were converted to IL-17-IFN- $\gamma^{+}$cells, so-called ex-Th17 cells, 


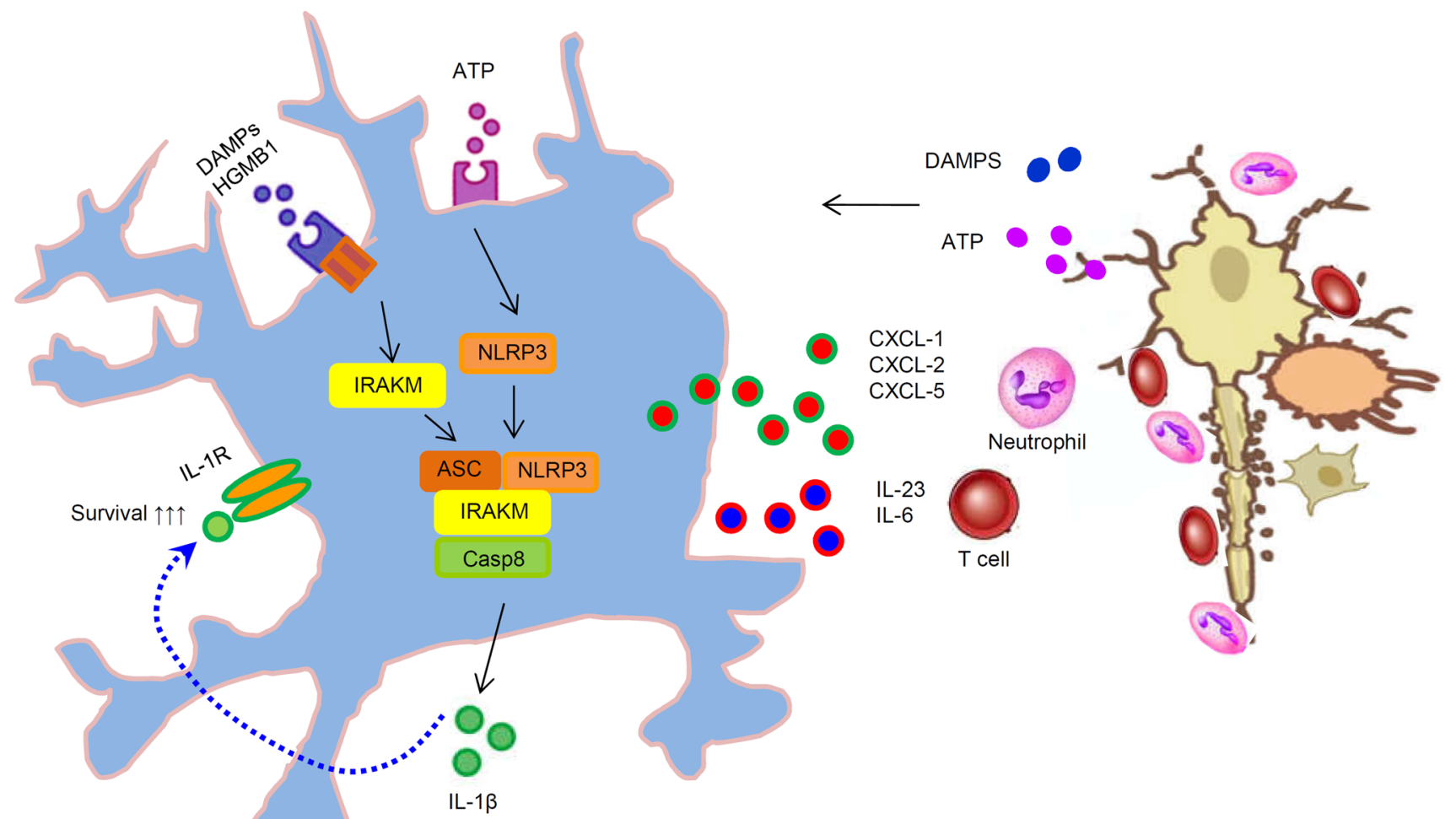

Figure 8. Model of microglia-intrinsic IRAKM-caspase-8 noncanonical inflammasome pathway in EAE/MS pathogenesis.

which actually became the dominant IFN- $\gamma$-producing cell population in the spinal cord (1). The fact that both IL-17- and IFN- $\gamma$ producing cell populations were reduced in the brain tissue of microglia-specific ASC- and IRAKM-deficient mice implicated a possible role of microglia for the recruitment and activation of Th17 cells and/or ex-Th17 cells in the CNS. Additionally, the neutrophils were also substantially reduced at day 10 in brain tissue of microglia-specific ASC- and IRAKM-deficient mice. These results suggest the critical impact of the IRAKM-ASC axis in microglia for the recruitment/activation of $\mathrm{T}$ cells and neutrophils during EAE pathogenesis (Figure 8). Notably, in addition to NLRP3, a recent study also reported the involvement of NLRC4 inflammasome activation in microglia and astrocytes during neuroinflammation induced by lysophosphatidyl choline (35). Future studies are required to investigate the possible involvement of this IRAKMcaspase- 8 axis in other inflammasomes such as NLRC4 contributing to neuroinflammation.

\section{Methods}

Mice. WT, CX3Cr1-Cre ${ }^{E R-E Y F P}, C X 3 C r 1^{G F P}, N_{L R P} 3^{-/}$, and Casp $8^{f / f l}$ on C57BL/6 mice were purchased from The Jackson Laboratory. ASC $C^{A / f l}$ mice were generated in our lab as previously reported (3). IRAKM $M^{f / f l}$ mice were generated in our lab (Figure 4, A and B). Casp $8^{-/-}$and Rip $3^{-/-}$ mice were a gift from William J. Kaiser, Department of Microbiology and Immunology, Emory Vaccine Center, Atlanta, Georgia, USA. Il1 $\beta^{--}$mice were a gift from Yoichiro Iwakura, Institute for Biomedical Sciences, Tokyo University of Science, Yamazaki, Noda, Japan. All the mice were on C57BL/6 background and maintained at Cleveland Clinic. Age- and sex-matched littermate control mice were used for each experiment.
Human brain and spinal cord specimens. The human brain and spinal cord specimens from patients with primary progressive multiple sclerosis were provided by Richard Reynolds, Imperial College London, London, United Kingdom.

Cell line. The human microglia cell line SV40 was provided by Theoharis C. Theoharides, Department of Integrative Physiology and Pathobiology, Tufts University School of Medicine, Boston, MA, USA. Plasmocin was used to rule out contamination during the cell culture.

Reagents. Anti-CD19 (1:400, 1D3) was from eBioscience. AntiCD45 (1:500, 30-F11), anti-F4/80 (1:200, BM8), anti-CD4 (1:200, L3T4), anti-Ly6C (1:300, HK1.4), anti-IFN- $\gamma$ (1:200, XMG1.2), antiIL-1R/CD121a (1:500, JAMA-147, 113505), lgG isotype control of IL-1R (1:500, HTK888), and ly6G (1:300, 1A8) were from BioLegend. AntiASC (22514-R, 04-147 Millipore), anti-NLRP3 (1:500, H-66), and actin ( $\beta$-actin antibody [C4], sc-47778) were purchased from Santa Cruz Biotechnology and Millipore. Anti-IL-1 $\beta$ (1:1,000, AF-401-NA), ELISA kit (IL-1b, IL-23, IL-6, CCL20, CXCL1, CXCL5), anti-IL-17A (1:300, 559502), anti-CD8 (1:300, 553041), Brdu (550891), anti-Ly6G (1:300, 1A8), and CD8 $(1: 300,553041)$ were purchased from BD Biosciences. Cleaved caspase-8 (9496 and 9429 from CST; ALX-804-448 from Enzo), P-IKBa (1:1,000, 2859), IKBa (1:1,000, 4812), cleaved caspase-1 (4199), IRAK1 (1:1,000, 4504), IRAK2 (1:1,000, 4367), and anti-human IL-1b (1:1,000, 2021) were from Cell Signaling Technology (CST). IRAKM (1:1,000, PAB7483) was from Abnova. Anti-caspase-1 (p20) mouse monoclonal antibody (AG-20B-0042) was from Adipogen. Anti-Ki67 (1:1,000, ab15580), anti-Brdu (ab1893, 1:200), and HMGB1 (ab81876 and ab181949) were from Abcam. Caspase-1/8 FLICA (97 and 99) were purchased from ImmunoChemistry. YVADfmk (ALX-260-154-R100) and IETD-fmk (550380) were purchased 
from Enzo and BD Biosciences. Luxol Fast Blue MBS Solution (26681) was purchased from Electron Microscopy Sciences. Protein A and G sepharose (17-046901, 17-061801) were from GE Healthcare. ATP (A1852) and TERM119 (1:200, HPA051870) were from Sigma-Aldrich. IBA1 (1:200, 019 19741) was from Wako. CPGB (ODN 2006 and ODN 1826) was from Invivogen. Escherichia coli LPS serotype O1101:B4 (201) was from List Biological Laboratories. IL-1Ra (Anakinra) was purchased from AMGEN.

Quantitative real-time PCR. EAE mice were euthanized and perfused with $1 \times$ PBS to remove the blood. The spinal cords were harvested and total RNA was extracted using TRIzol reagent according to the manufacturer's instructions (Invitrogen). The cDNA was synthesized with Oligo (dT) primers and superscript II reverse transcriptase (Invitrogen) by using $1 \mu \mathrm{g}$ total RNA. The cDNA was resuspended in $100 \mu \mathrm{l}$ $\mathrm{H}_{2} \mathrm{O}$, and $2 \mu \mathrm{l}$ cDNA samples were used for real-time PCR in a total volume of $20 \mu$ l SYBR Green reagent (Invitrogen) and specific primers. All gene expression results are expressed as arbitrary units relative to the expression of $\beta$-actin. The following real-time PCR (RT-PCR) primers were used for mouse genes: IL-17A, $5^{\prime}$-gtccagggagagcttcatctg- $3^{\prime}$ and $5^{\prime}$-cttggcetcagtgtttggac- $3^{\prime}$; IFN- $\gamma, 5^{\prime}$-ctcatggctgtttctggctg- $3^{\prime}$ and $5^{\prime}$-ccttttgccagttcctccag- $3^{\prime} ; \quad$ TNF- $\alpha, \quad 5^{\prime}$-ccaccacgctcttctgtcta- $3^{\prime}$ and $5^{\prime}$-gatctgagtgtgagggtctgg- $3^{\prime} ; \mathrm{Ccl} 20,5^{\prime}$-cacaagacagatggccgatg- $3^{\prime}$ and $5^{\prime}$-cccttttcacccagttctgc-3'; Cxcl1, $5^{\prime}$-ccagagcttgaaggtgttgc- $3^{\prime}$ and $5^{\prime}$-tgaaccaagggagcttcagg- $3^{\prime} ;$ IL-1b, 5'-ccatcctctgtgactcatggg- $3^{\prime}$ and $5^{\prime}$-tcagctcatatgggtccgac-3'; IL-6, 5'-GACAAAGCCAGAGTCCTTCAGAGAG-3' and $5^{\prime}$-CTAGGTTTGCCGAGTAGATCTC-3'; $\beta$-actin, 5'-AGATGTGGATCAGCAAGCAG-3' and 5'-GCGCAAGTTAGGTTTTGTCA-3'; IL-18, 5' -gccgacttcactgtacaacc-3' and 5'-gtctggtctggggttcactg-3'; IL-23/p19, 5'-TCCCTACTAGGACTCAGCCAACTC-3' and $5^{\prime}$-ACTCAGGCTGGGCACTG-3'; STEAP4, 5' -gaacactagatgcaagccaa- $3^{\prime}$ and $5^{\prime}$-gagagatccttggtccagtgg- $3^{\prime}$.

Cell surface and intracellular staining. For cell surface staining, single-cell suspensions were incubated with Fc blocking buffer (Fc block diluted in FACS buffer at 1:50 ratio) on ice for 30 minutes at $4^{\circ} \mathrm{C}$. After washing, the cells were stained with cell surface antibody for $30 \mathrm{~min}$ utes at $4^{\circ} \mathrm{C}$. For intracellular staining, single-cell suspensions were cultured for 5 hours with phorbol 12-myristate 13-acetate (PMA, 20 $\mathrm{ng} / \mathrm{ml}$, MilliporeSigma) plus ionomycin (500 ng/ml, MilliporeSigma). GolgiStop (1:500, BD Biosciences) was added during the final 2 hours of incubation. After washing, cells were fixed by fixation/permeabilization solution (BD Cytofix/Cytoperm Kit, BD Biosciences) according to the manufacturer's instructions. After incubation with Perm/Wash buffer (BD Biosciences) for 1 hour, antibodies for intracellular staining were added for 1 hour in $4^{\circ} \mathrm{C}$ and cells were analyzed on a FACSCalibur (BD Biosciences).

Primary microglia culture. Adult 6-week-old mice were euthanized and perfused with PBS to remove the blood, followed by brain collection and digestion with the Adult Brain Dissociation Kit (130-107-677, MACS) following the manufacturer's instructions. The digested brain was homogenized and primary microglia were isolated by a discontinuous Percoll gradient. Isolated microglia were seeded on the plate and cultured with DMEM/F12 supplemented with $100 \mathrm{U} / \mathrm{ml}$ penicillin and streptomycin, 10\% FBS, and $5 \mathrm{ng} / \mathrm{ml}$ mouse GM-CSF (415-ML/CF, R\&D Systems) After 14 days, microglia were harvested by collecting the culture media and centrifuging for 5 minutes at $412 \mathrm{~g}$. The purity of the microglia were confirmed by flow cytometry analysis after being stained with antibodies against CD 45 and CD11b (>99\% purity).
Generation of chimeric mice. The chimera mice were generated as previously reported (36). Briefly, 6-week-old recipient mice were subjected to 9 Gy of total body lethal irradiation and allowed for 4 hours of recovery before reconstitution with bone marrow cells. Sixweek-old donor mice were sacrificed by $\mathrm{CO}_{2}$ asphyxiation and bone marrow cells were flushed from femurs and tibias. The recipient mice were transferred with $1.5 \times 10^{7}$ WT bone marrow cells in $150 \mu \mathrm{l} 1 \times \mathrm{PBS}$ intravenously through retro-orbital venous sinus. Mice were fed with gentamicin-containing $(10 \mu \mathrm{g} / \mathrm{ml})$ drinking water for an additional 4 weeks before subjected to MOG immunization.

Tamoxifen injection. Tamoxifen-induced Cre expression was performed as previously reported (2) (Supplemental Figure 1A). One week before bone marrow transplantation, tamoxifen was administered i.p. at $5 \mathrm{mg} / \mathrm{mouse}$ for 4 consecutive weeks (once per week). EAE was induced after tamoxifen treatment (4 weeks after bone marrow transplantation).

Induction and assessment of EAE. Active EAE and adoptive transfer (passive) EAE were induced and assessed as previously described by our group $(2,3,37)$. Briefly, for the adoptive transfer, donor mice were immunized with $\mathrm{MOG}_{35-55}$ subcutaneously and spleen/draining lymph nodes were harvested 10 days after immunization. The cells were cultured for 5 days with $\mathrm{MOG}_{35-55}$ at a concentration of $25 \mu \mathrm{g} /$ $\mathrm{ml}$ under either Th1 cell-polarizing conditions (20 ng/ml IL-12, R\&D Systems; $2 \mu \mathrm{g} / \mathrm{ml}$ anti-IL-23p19, eBiosciences) or Th17 cell-polarizing conditions (20 ng/ml IL-23, R\&D Systems). We injected recipient mice with $3.0 \times 10^{7}$ polarized $\mathrm{MOG}_{35-55}$-specific Th1 or Th17 cells 4 hours after exposing them to 5 Gy sublethal irradiation. Clinical scores were assessed in a double-blinded manner every other day.

BrdU incorporation assay. The animals were administrated BrdU (50 $\mathrm{mg} / \mathrm{kg}$, once per day) for 3 consecutive days by i.p. injection. Brains and spinal cords were fixed with $4 \%$ PFA overnight followed by dehydration with $15 \%$ and $30 \%$ sucrose for an additional 2 days. Tissues were quickly frozen in OCT and cut as $20 \mu \mathrm{m} / \mathrm{slice}$. Tissue sections were treated with $2 \mathrm{M}$ hydrochloric acid for 30 minutes at room temperature followed by treatment with $0.01 \mathrm{M}$ borate buffer ( $\mathrm{pH}$ 8.2) for 30 minutes at room temperature. Slices were then subjected to immunohistochemistry analysis.

Intracerebroventricular injection of microglia. Microglia injection was performed as previously reported (22). Briefly, microglia isolated from WT or $\mathrm{IL}^{-1 \mathrm{~b}^{-/-}}$adult naive mice were injected $\left(5.5 \times 10^{5}\right.$ in $4 \mu \mathrm{l}$ PBS per mouse) into the cerebrospinal fluid (mediolateral, $-0.8 \mathrm{~mm}$; anteroposterior, $-0.4 \mathrm{~mm}$; dorsoventral, $-2.5 \mathrm{~mm}$ ) of $\mathrm{Csf} 1 \mathrm{r}^{f l / f l} \mathrm{Cx} 3 \mathrm{cr} 1^{\text {cre }}$ mice after Cre induction by tamoxifen injection followed by immunization with $\mathrm{MOG}_{35-55}$ peptide.

Histological analysis. EAE mice were perfused with PBS and PFA before harvesting spinal cords. The spinal cord was quick-frozen in OCT and cut to approximately $10-$ to $20-\mu \mathrm{m}$ thickness. Sections were stained with H\&E and with Luxol Fast Blue (Electron Microscope Sciences) for evaluation of inflammation and demyelination, respectively.

Enzyme-linked immunosorbent assay (ELISA). The media from cultured microglia was harvested and centrifuged. The supernatant was subjected to ELISA (all kits from R\&D Systems) according to the manufacturer's instructions. The concentration of cytokines in the media was adjusted based on the standard curve.

Immunofluorescence staining. Immunofluorescence staining was performed as previously reported (2). Briefly, sections were washed and blocked, followed by incubating with first antibodies overnight at $4^{\circ} \mathrm{C}$. After washing and incubating with a second antibody for 1 
hour at room temperature, the slices were either stained with DAPI or mounted for the microscope. All images were captured with a confocal microscope (Artemis or Keyence BZ-X710).

Immunoblotting and immunoprecipitation. Cells were lysed in lysis buffer (0.5\% Triton X-100, 20 mM HEPES, pH 7.4, 150 mM NaCl, 12.5 $\mathrm{mM} \beta$-glycerophosphate, $1.5 \mathrm{mM} \mathrm{MgCl}, 10 \mathrm{mM} \mathrm{NaF}, 2 \mathrm{mM}$ dithiothreitol, $1 \mathrm{mM}$ sodium orthovanadate, $2 \mathrm{mM}$ EGTA, $20 \mathrm{mM}$ aprotinin, $1 \mathrm{mM}$ phenylmethylsulfonyl fluoride). Protein lysate $(20 \mu \mathrm{g})$ was run per lane on an approximately $8 \%-12 \%$ SDS-PAGE gel and subjected to immunoblotting with different antibodies. Coimmunoprecipitation experiments were performed as previously described $(2,38)$. To avoid the heavy chain band, we used a different source (such as immunoprecipitation with rabbit-derived antibody and probe with mouse-derived antibody) to target the same protein.

Statistics. The $P$ values of clinical scores were determined by 2-way multiple-range ANOVA for multiple comparisons. Other $P$ values were determined by unpaired 2-tailed Student's $t$ test. All results are mean \pm SEM. ${ }^{\star} P<0.05 ;{ }^{* *} P<0.01 ;{ }^{* *} P<0.001 ;{ }^{* * *} P<$ 0.0001. All data were from at least 2 independent experiments.

Study approval. All animal experiments were conducted and approved by the Cleveland Clinic Institutional Animal Care and Use Committee. The human tissue experiments were approved by the ethics committees at Imperial College London and the Cleveland Clinic. Written informed consent was received from participants prior to inclusion in the study.

\section{Author contributions}

$\mathrm{XL}$ and CJZ conceived and coordinated the study, and wrote the manuscript. CJZ conducted the majority of experiments and analyzed the data. MJ, WL, QZ, and CW helped with experiments. XC, JX, BH, and AF provided technical support. HZ, JG, JC, and AD performed the mass spectometry and analyzed the data. ZK, WJK, MAM, YI, GD, DWA, FDS, and RMR provided critical discussion.

\section{Acknowledgments}

This investigation was supported by grants from the National Multiple Sclerosis Society (RG-1707-28180 and RG5130A2/1 to XL) and National Institutes of Health (5R01NS071996-05, 1RO1AA023722, and MSTP-T32GM007250 to XL). The study was partially supported by a grant from the National Science Foundation of China (81701235 to CZ).

Address correspondence to: Cun-Jin Zhang, Medical School of Nanjing University, No. 22 Hankou Road, Nanjing, Jiangsu 210093 China. Phone: 86.222.354.2851; Email: zhangcunjin516@163.com. Or to: Xiaoxia Li, Department of Immunology, Cleveland Clinic Lerner Research Institute, 9500 Euclid Avenue, NE4-256, Cleveland, Ohio 44195, USA. Phone: 216.445.8706; Email: lix@ccf.org.
1. Hirota K, et al. Fate mapping of IL-17-producing $\mathrm{T}$ cells in inflammatory responses. Nat Immunol. 2011;12(3):255-263.

2. Wang C, et al. IL-17 induced NOTCH1 activation in oligodendrocyte progenitor cells enhances proliferation and inflammatory gene expression. Nat Commun. 2017;8:15508

3. Martin BN, et al. T cell-intrinsic ASC critically promotes $\mathrm{T}(\mathrm{H}) 17$-mediated experimental autoimmune encephalomyelitis. Nat Immunol. 2016;17(5):583-592.

4. Fletcher JM, Lalor SJ, Sweeney CM, Tubridy N, Mills KH. T cells in multiple sclerosis and experimental autoimmune encephalomyelitis. Clin Exp Immunol. 2010;162(1):1-11.

5. Rothhammer V, et al. Th17 lymphocytes traffic to the central nervous system independently of $\alpha 4$ integrin expression during EAE. J Exp Med. 2011;208(12):2465-2476.

6. Iwakura Y, Ishigame H. The IL-23/IL-17 axis in inflammation. JClin Invest. 2006;116(5):1218-1222.

7. Lévesque SA, et al. Myeloid cell transmigration across the CNS vasculature triggers IL-1 $\beta$-driven neuroinflammation during autoimmune encephalomyelitis in mice. JExp Med. 2016;213(6):929-949.

8. Lin CC, Edelson BT. New insights into the role of IL-1 $\beta$ in experimental autoimmune encephalomyelitis and multiple sclerosis. Jimmunol. 2017;198(12):4553-4560.

9. Ronchi F, et al. Experimental priming of encephalitogenic Th1/Th17 cells requires pertussis toxindriven IL-1 $\beta$ production by myeloid cells. Nat Commun. 2016;7:11541.

10. Burm SM, et al. Expression of IL-1 $\beta$ in rhesus EAE and MS lesions is mainly induced in the CNS itself. J Neuroinflammation. 2016;13(1):138.

11. Aglietti RA, et al. GsdmD p30 elicited by caspase-11 during pyroptosis forms pores in membranes. Proc Natl Acad Sci USA. 2016;113(28):7858-7863.

12. He WT, et al. Gasdermin D is an executor of pyroptosis and required for interleukin- $1 \beta$ secretion. Cell Res. 2015;25(12):1285-1298.

13. Shi J, et al. Cleavage of GSDMD by inflammatory caspases determines pyroptotic cell death. Nature. 2015;526(7575):660-665.

14. Ding J, et al. Pore-forming activity and structural autoinhibition of the gasdermin family. Nature. 2016;535(7610):111-116.

15. Boucher D, et al. Caspase-1 self-cleavage is an intrinsic mechanism to terminate inflammasome activity. J Exp Med. 2018;215(3):827-840.

16. Inoue M, Shinohara ML. NLRP3 inflammasome and MS/EAE. Autoimmune Dis. 2013;2013:859145.

17. Inoue M, Williams KL, Gunn MD, Shinohara ML. NLRP3 inflammasome induces chemotactic immune cell migration to the CNS in experimental autoimmune encephalomyelitis. Proc Natl Acad Sci U S A. 2012;109(26):10480-10485.

18. Shaw PJ, Lukens JR, Burns S, Chi H, McGargill MA, Kanneganti TD. Cutting edge: critical role for PYCARD/ASC in the development of experimental autoimmune encephalomyelitis. J Immunol. 2010;184(9):4610-4614.

19. Chang M, Jin W, Sun SC. Peli1 facilitates TRIF-dependent Toll-like receptor signaling and proinflammatory cytokine production. Nat Immunol. 2009;10(10):1089-1095.

20. Biber K, Möller T, Boddeke E, Prinz M. Central nervous system myeloid cells as drug targets: current status and translational challenges. Nat Rev Drug Discov. 2016;15(2):110-124.

21. Burguillos MA, et al. Caspase signalling controls microglia activation and neurotoxicity. Nature.
2011;472(7343):319-324.

22. Xiao Y, et al. Peli1 promotes microglia-mediated CNS inflammation by regulating Traf3 degradation. Nat Med. 2013;19(5):595-602.

23. Monif M, Reid CA, Powell KL, Smart ML, Williams DA. The $\mathrm{P} 2 \mathrm{X} 7$ receptor drives microglial activation and proliferation: a trophic role for P2X7R pore. J Neurosci. 2009;29(12):3781-3791.

24. Chastain EM, Duncan DS, Rodgers JM, Miller SD. The role of antigen presenting cells in multiple sclerosis. Biochim Biophys Acta. 2011;1812(2):265-274.

25. Kivisäkk P, et al. Localizing central nervous system immune surveillance: meningeal antigen-presenting cells activate T cells during experimental autoimmune encephalomyelitis. Ann Neurol. 2009;65(4):457-469.

26. Goverman J. Autoimmune T cell responses in the central nervous system. Nat Rev Immunol. 2009;9(6):393-407.

27. Antonopoulos C, El Sanadi C, Kaiser WJ, Mocarski ES, Dubyak GR. Proapoptotic chemotherapeutic drugs induce noncanonical processing and release of IL- $1 \beta$ via caspase- 8 in dendritic cells. JImmunol. 2013;191(9):4789-4803.

28. Gringhuis SI, et al. Dectin-1 is an extracellular pathogen sensor for the induction and processing of IL- $1 \beta$ via a noncanonical caspase- 8 inflammasome. Nat Immunol. 2012;13(3):246-254.

29. Bossaller L, et al. Cutting edge: FAS (CD95) mediates noncanonical IL-1 $\beta$ and IL-18 maturation via caspase- 8 in an RIP3-independent manner. JImmunol. 2012;189(12):5508-5512.

30. Ajami B, Bennett JL, Krieger C, McNagny KM, Rossi FM. Infiltrating monocytes trigger EAE progression, but do not contribute to the resident microglia pool. Nat Neurosci. 2011;14(9):1142-1149.

31. Ding Z, et al. Antiviral drug ganciclovir is a potent 
inhibitor of microglial proliferation and neuroinflammation. JExp Med. 2014;211(2):189-198.

32. Bruttger J, et al. Genetic cell ablation reveals clusters of local self-renewing microglia in the mammalian central nervous system. Immunity. 2015;43(1):92-106.

33. Ofengeim $D$, et al. Activation of necroptosis in multiple sclerosis. Cell Reports. 2015;10(11):1836-1849.

34. Viceconte N, Burguillos MA, Herrera AJ, De Pablos RM, Joseph B, Venero JL. Neuromelanin activates proinflammatory microglia through a caspase-8-dependent mechanism. J Neuroinflammation. 2015;12:5.

35. Freeman L, Guo H, David CN, Brickey WJ, Jha S, Ting JP. NLR members NLRC4 and NLRP3 mediate sterile inflammasome activation in microglia and astrocytes. JExp Med. 2017;214(5):1351-1370.

36. Cardona AE, et al. Scavenging roles of chemokine receptors: chemokine receptor deficiency is asso- ciated with increased levels of ligand in circulation and tissues. Blood. 2008;112(2):256-263.

37. Qian Y, et al. The adaptor Act1 is required for interleukin 17-dependent signaling associated with autoimmune and inflammatory disease. Nat Immunol. 2007;8(3):247-256.

38. Wang C, et al. The psoriasis-associated D1ON variant of the adaptor Act1 with impaired regulation by the molecular chaperone hsp90. Nat Immunol.2013;14(1):72-81. 